\title{
PTCH1 pathway network model in diffuse-type gastric cancer and epithelial mesenchymal transition
}

\author{
Shihori Tanabe ${ }^{1 *}$, Kazuhiko Aoyagi ${ }^{2}$, Hiroshi Yokozaki ${ }^{3}$ and Hiroki Sasaki ${ }^{4}$ \\ ${ }^{1}$ Division of Risk Assessment, Biological Safety Research Centre, National Institute of Health Sciences, Japan \\ ${ }^{2}$ Department of Clinical Genomics, National Cancer Centre Research Institute, Japan \\ ${ }^{3}$ Department of Pathology, Kobe University of Graduate School of Medicine, Japan \\ ${ }^{4}$ Department of Translational Oncology, FIOC, National Cancer Centre Research Institute, Japan
}

\begin{abstract}
Patched 1 (PTCH1) gene plays an important role in the Hedgehog signalling in cancer. To reveal the role of PTCH1 and the network in epithelial mesenchymal transition (EMT), gene expression and molecular network of the PTCH1 was investigated in mesenchymal stem cells (MSCs) and diffuse-type gastric cancer (GC). The PTCH1 gene expression was up-regulated in diffuse-type GC compared to MSCs. PTCH1 network model was generated with the gene expression profiling of the molecules related to PTCH1 and EMT. The signalling and molecular network of PTCH1 was analyzed using several databases, including cBioPortal for Cancer Genomics, Kyoto Encyclopedia of Genes and Genomes (KEGG), BioGRID, VaProS and Ingenuity Pathways Analysis (IPA) databases. The PTCH1 model network contains cancer-related genes such as cadherin 1 (CDH1), catenin beta 1 (CTNNB1) and transforming growth factor beta receptor 3 (TGFBR3). The results revealed a PTCH1 pathway network model in cancer and stem cells.
\end{abstract}

\section{Introduction}

The Patched (PTCH) protein is the receptor for Sonic Hedgehog (Shh) and functions in tumorigenesis and the development of embryonic structures through inhibiting cancer-related pathways by the inhibition of the signalling of the seven-transmembrane protein Smoothened (SMO) [1]. It has been shown that the binding of Shh to Patched 1 (PTCH1) prevents its inhibition of SMO [1]. SMO, gliomaassociated oncogene 1 (GLI1), and forkhead box M1 (FOXM1), which are implicated in tumour formation, are up-regulated in cervical cancer tissues and are correlated with the clinical stage of tumours, whereas the expression of Shh, PTCH1 and GLI1 proteins is correlated with the pathological grade [2]. Internalization and degradation of PTCH1 is regulated by Itchy homolog (Itch), E3 ubiquitin-protein ligase, and the C-terminal domain of PTCH1, leading to Gli activation and cell death [3]. The Sonic Hedgehog pathway consists of SHH, PTCH1, SMO, Gli1, Gli2, Gli3 and regulates the transcription of Gli1, PTCH1, Cyclin D1, c-Myc and Bcl-2 [4]. The Hedgehog (Hh) signalling pathway and p53-mediated DNA repair mechanisms are dysregulated in human cancer, and this dysregulation is associated with the PTCH1 homolog, PTCH53, activated by p53 [5]. The expression of Hh signalling pathway molecules in articular cartilage during adjuvant-induced arthritis is up-regulated, which provides justification for the clinical implication of Hh signalling [6]. The micro-RNA miRNA-206 was found to be down-regulated in the Shh subgroup of medulloblastoma in analysis of Smo+/+ transgenic mice and Ptch1+/- knockout mice, which suggests the role of miRNAs in tumour suppression [7].

Epithelial-mesenchymal transition (EMT) is the phenotypic shift from epithelial phenotype into mesenchymal phenotype of the cells, which contributes to the cancer metastasis and malignancy. EMT occurs in cancer development and the EMT contributes into cancer stem cell (CSC) maintenance [8]. To investigate the role of EMT in
Shh pathways in cancer, we compared gene expression of PTCH1 in mesenchymal stem cells (MSCs) and diffuse-type gastric cancer (GC). MSCs are stem cells with mesenchymal features and diffuse-type GC exhibits more mesenchymal-like features compared to intestinal-type GC. It may be possible to reveal the EMT-related molecular networks by comparing MSCs and diffuse-type GC. We have previously shown the involvement of catenin beta 1 (CTNNB1) in diffuse-type GC compared to MSCs [9]. During the process of database analysis with cBioPortal for Cancer Genomics, PTCH1 was found to be co-altered with CTNNB1 in stomach adenocarcinoma. It is known that tissuespecific accumulation of genetic mutations in human adult stem cells is an important factor for disease prognosis [10]. In this article, we focused on the regulation and role of PTCH1 in stem cells and cancer.

\section{Materials and methods}

\section{Gene expression analysis of MSCs and GC}

The gene expression in MSCs (6 early- and 6 late- stage cultures, $\mathrm{n}=12)$ and diffuse-type GC $(\mathrm{n}=5)$ was analysed with GeneChip Human Genome U133 Plus 2.0 microarray (Affymetrix, Santa Clara, California, USA), as previously described $[9,11,12]$. Briefly, total RNA purified from the cells were biotinylated and hybridized to the microarray. The signal intensity in each gene was analysed and

${ }^{*}$ Correspondence to: Shihori Tanabe, Division of Risk Assessment, Biological Safety Research Center, National Institute of Health Sciences, 3-25-26, Tonomachi, Kawasaki-ku, Kawasaki, 210-9501, Japan, Tel: 81-44-270-6686, Fax: 81-44-270-6703, E-mail: stanabe@nihs.go.jp

Key words: EMT, gastric cancer, MSC, PTCH1, stem cell

Received: August 10, 2018; Accepted: August 23, 2018; Published: August 28 2018 
compared between MSCs and diffuse-type GC. The microarray data for MSCs and diffuse-type GC are available to the public in NCBI's Gene Expression Omnibus (GEO) database and are accessible via GEO Series accession number GSE7888 (https://www.ncbi.nlm.nih.gov/geo/ query/acc.cgi?acc=GSE7888) and GSE42252 (https://www.ncbi.nlm. nih.gov/geo/query/acc.cgi?acc=GSE42252), respectively $[9,11,12]$.

\section{Cell culture}

Human MSCs from bone marrow (Lonza, Walkersville, MD, USA) were cultured in MSC growth medium (MSCGM; Lonza \#PT-3001; MSC basal medium supplemented with mesenchymal cell growth supplement, $\mathrm{L}$-glutamine and penicillin/streptomycin) at $37^{\circ} \mathrm{C}$ in a $\mathrm{CO}_{2}$ (5\%) incubator. Cells were passaged according to the manufacturer's protocol; however a slight modification was made to use trypsin-EDTA solution (Lonza \#CC-3232). Lot numbers of the human MSC batches were as follows: \#4F1127, \#4F0312, \#5F0138, \#4F1560, \#4F0591 and \#4F0760. Informed consent was obtained for Poietics human MSC systems (Lonza) [12]. Passage numbers of MSC cultures were \#4 and \#22 for \#4F1127, \#4 and \#28 for \#4F0312, \#4 and \#24 for \#5F0138, $\# 5$ and \#28 for \#4F1560, \#4 and \#28 for \#4F0591, and \#4 and \#28 for \#4F0760.

\section{Diffuse-type GC tissues}

Diffuse-type GC tissues were originally provided by the National Cancer Centre Hospital after obtaining written informed consent from each patient and approval from the National Cancer Centre Institutional Review Board. All cancer specimens were reviewed and classified histopathologically according to the Japanese Classification of Gastric Cancer. Following surgical extraction, tissue specimens were immediately frozen with liquid nitrogen and stored at $-80^{\circ} \mathrm{C}$ until microarray analysis $[9,11]$. Existing, publicly available data were analysed in the article.

\section{Analysis with cBioPortal for cancer genomics}

The cancergenomics data analysis related to CTNNB1 wasperformed using cBioPortal for Cancer Genomics (http://www.cbioportal.org) $[13,14]$. The term "PTCH1" was searched in the cBioPortal for Cancer Genomics, and the cross-cancer alteration summary for PTCH1 was obtained. Studies of stomach adenocarcinoma (TCGA, Nature 2014) were further analysed for pathway network analysis [15].

\section{Gene ontology analysis of PTCH1}

Gene Ontology of PTCH1 was analysed using several databases, including EMBL-EBI (http://www.ebi.ac.uk/QuickGO/), AmiGO 2 (http://amigo.geneontology.org/amigo/landing) and the Gene Ontology Consortium (http://geneontology.org/).

\section{Pathway network analysis of PTCH1 and related genes}

Pathway network analysis was performed using the databases VaProS (http://pford.info/vapros/), KEGG (http://www.genome.jp/ kegg/), cBioPortal for Cancer Genomics (http://www.cbioportal.org), and Cytoscape (http://www.cytoscape.org/). Molecular interactions were analysed using the BioGRID (http://www.thebiogrid.org) database [16]. Molecule localization was analyzed using The Human Protein Atlas (http://www.proteinatlas.org/) [17] and UniProt (http:// www.uniprot.org/) [18]. The NextBio (http://www.nextbio.com/b/ nextbio.nb) was used for the exploration of the gene expression in gastric cancer [19].

\section{Network generation}

The list of PTCH1 and EMT-related genes was uploaded in the Ingenuity Pathway Analysis (IPA, https://analysis.ingenuity.com/pa/ launch.jsp). Each identifier was mapped to its corresponding object in Ingenuity's Knowledge Base [20].

\section{Statistical analysis}

The data were expressed as the mean \pm SE. Student's t-test was performed in Microsoft Excel (Microsoft, Redmond, WA, USA). $p<$ 0.001 ( $n=12$ in MSCs, $n=5$ in diffuse-type GC) was considered as statistically significant.

\section{Results}

\section{Gene expression of PTCH1 and EMT-related genes in MSCs and diffuse-type GC}

The gene expression of PTCH1 was up-regulated in diffuse-type GC compared to MSCs as shown in figure 1. The gene expression of PTCH1 was stably low after each passage of MSCs, whereas the gene expression of PTCH1 in diffuse-type GC was up-regulated. Notably, in one of the diffuse-type GC samples, PTCH1 gene expression was not altered. Gene expression of vimentin (VIM), an EMT-related gene, was up-regulated in one probe set and down-regulated in another probe set in diffuse-type GC compared to MSCs [11]. On the other hand, gene expression of twist family bHLH transcription factor 1 (TWIST1) and snail family transcriptional repressor 2 (SNAI2) were down-regulated in diffuse-type GC compared to MSCs [9,21]. Other EMT-related genes, TWIST2, SNAI1 or actin, alpha 1, skeletal muscle (ACTA1) had low signal intensity. Gene expression of GLI family zinc finger 3 (GLI3) was not changed between MSCs and diffuse-type GC. From the immunohistochemistry data in the Human Protein Atlas, E-cadherin (CDH1), of which gene expression was down-regulated in MSCs compared to diffuse-type GC, was less expressed in progressed cancer [11].

\section{Gene ontology of PTCH1}

According to KEGG, the gene ontology of PTCH1 is as follows: cAMP signalling pathway, Hedgehog signalling pathway, axon guidance, pathways in cancer, proteoglycans in cancer, and basal cell carcinoma. Genes in which the GO terms include regulation of EMT (GO:0010717) have been analysed with AmiGO 2 and are shown in table 1 (http://amigo.geneontology.org/amigo/term/GO:0010717).

\section{Pathway network analysis of PTCH1 and EMT-related genes}

According to the analysis of cBioPortal for Cancer Genomics, PTCH1 interacts with GLI3 in the network generated using stomach adenocarcinoma samples (TCGA, Nature 2014) (Figure 2) [15]. Next, the network was generated with PTCH1 and EMT-related genes. Information and the gene expression of 89 input genes, including PTCH1, from cBioPortal for Cancer Genomics are listed in table 2. The network contained 139 nodes and included the 89 query genes, and the most frequently altered neighbour genes (out of a total of 3060) in cBioPortal. The network includes 66 core genes originally queried and neighbouring genes. From the analysis of molecular interactions in VaProS, PTCH1 was found to be associated with the TGFB2 network. PTCH1 is also associated with TGFBR3 and TGFB2 in the VaProS molecular network.

Among the genes in the network generated with cBioPortal, the queried core genes that are connected with other core genes were 
Table 1. Molecules related to the regulation of EMT (AmiGO2, Homo sapiens)

\begin{tabular}{|c|c|c|c|}
\hline Gene Symbol & Gene/ product name & Contributor & Reference \\
\hline ACVR1 & Activin receptor type-1 & BHF-UCL & GO_REF:0000024 \\
\hline ADIPOR1 & Adiponectin receptor protein 1 & BHF-UCL & PMID:23776679 \\
\hline AGER & Advanced glycosylation end product-specific receptor & Ensembl & GO_REF:0000019 \\
\hline ALX1 & ALX homeobox protein 1 & UniProt & PMID:23288509 \\
\hline AXIN2 & Axin-2 & BHF-UCL & PMID: 17072303 \\
\hline BAMBI & BMP and activin membrane-bound inhibitor homolog & BHF-UCL & PMID: 19328798 \\
\hline BCL9L & B-cell CLL/lymphoma 9-like protein & BHF-UCL & PMID: 19328798 \\
\hline BMP2 & Bone morphogenetic protein 2 & BHF-UCL & PMID:20890042 \\
\hline BMP5 & Bone morphogenetic protein 5 & BHF-UCL & PMID:21319131 \\
\hline CLASP1 & CLIP-associating protein 1 & UniProt & PMID:23940118 \\
\hline CLASP2 & CLIP-associating protein 2 & UniProt & PMID:23940118 \\
\hline COL1A1 & Collagen type I alpha-1 chain & UniProt & PMID:20018240 \\
\hline CRB2 & Protein crumbs homolog 2 & UniProt & GO_REF:0000024 \\
\hline CTNNB1 & Catenin beta-1 & MGI & PMID:23983127 \\
\hline DAB2 & Disabled homolog 2 & UniProt & PMID: 15734730 \\
\hline DAB2IP & Disabled homolog 2-interacting protein & BHF-UCL & PMID:20080667 \\
\hline DACT3 & Dapper homolog 3 & Ensembl & GO_REF:0000019 \\
\hline DAG1 & Dystroglycan & UniProt & PMID:23940118 \\
\hline EFNA1 & Ephrin-A1 & BHF-UCL & GO_REF:0000024 \\
\hline ELL3 & RNA polymerase II elongation factor ELL3 & UniProt & GO_REF:0000024 \\
\hline ENG & Endoglin & BHF-UCL & GO_REF:0000024 \\
\hline EPB41L5 & Band 4.1-like protein 5 & Ensembl & GO_REF:0000019 \\
\hline EPHA3 & Ephrin type-A receptor 3 & UniProt & GO_REF:0000024 \\
\hline EZH2 & Histone-lysine N-methyltransferase EZH2 & UniProt & PMID:20154697 \\
\hline FAM195B & Mapk-regulated corepressor-interacting protein 1 & UniProt & PMID:25728771 \\
\hline FOXA1 & Hepatocyte nuclear factor 3-alpha & BHF-UCL & PMID:20160041 \\
\hline FOXA2 & Hepatocyte nuclear factor 3-beta & BHF-UCL & PMID:20160041 \\
\hline FUZ & Protein fuzzy homolog & UniProt & GO_REF:0000024 \\
\hline GCNT2 & $\mathrm{N}$-acetyllactosaminide beta-1,6-N-acetylglucosaminyl-transferase & UniProt & PMID:21750175 \\
\hline GLIPR2 & Golgi-associated plant pathogenesis-related protein 1 & UniProt & PMID:23516513 \\
\hline GREM1 & Gremlin-1 & UniProt & PMID:16816361 \\
\hline HDAC2 & Histone deacetylase 2 & Ensembl & GO_REF:0000019 \\
\hline HPN & Serine protease hepsin & UniProt & PMID:19843851 \\
\hline LDLRAD4 & Low-density lipoprotein receptor class A domain-containing protein 4 & UniProt & PMID:24627487 \\
\hline LEF1 & Lymphoid enhancer-binding factor 1 & UniProt & PMID:20018240 \\
\hline MAD2L2 & Mitotic spindle assembly checkpoint protein MAD2B & BHF-UCL & PMID:19443654 \\
\hline NKX2-1 & Homeobox protein $\mathrm{Nkx}-2.1$ & UniProt & PMID:19293183 \\
\hline NOTCH1 & Neurogenic locus notch homolog protein 1 & BHF-UCL & PMID:17984306 \\
\hline OLFM1 & Noelin & AgBase & GO_REF:0000024 \\
\hline OVOL2 & Transcription factor Ovo-like 2 & UniProt & GO_REF:0000024 \\
\hline PBLD & Phenazine biosynthesis-like domain-containing protein & UniProt & PMID:23687415 \\
\hline PHLDB1 & Pleckstrin homology-like domain family B member 1 & UniProt & PMID:23940118 \\
\hline PHLDB2 & Pleckstrin homology-like domain family B member 2 & UniProt & PMID:23940118 \\
\hline POFUT2 & GDP-fucose protein O-fucosyltransferase 2 & Ensembl & GO_REF:0000019 \\
\hline PPP2CA & Serine/threonine-protein phosphatase $2 \mathrm{~A}$ catalytic subunit alpha isoform & BHF-UCL & PMID:20080667 \\
\hline RGCC & Regulator of cell cycle RGCC & BHF-UCL & PMID:19158077 \\
\hline SDCBP & Syntenin-1 & UniProt & PMID:25893292 \\
\hline SDHAF2 & Succinate dehydrogenase assembly factor 2 , mitochondrial & MGI & PMID:23983127 \\
\hline SERPINB3 & Serpin B3 & UniProt & PMID:20527027 \\
\hline SFRP1 & Secreted frizzled-related protein 1 & UniProt & PMID:19095296 \\
\hline SFRP2 & Secreted frizzled-related protein 2 & UniProt & PMID:19095296 \\
\hline SMAD2 & Mothers against decapentaplegic homolog 2 & BHF-UCL & GO_REF:0000024 \\
\hline SMAD3 & Mothers against decapentaplegic homolog 3 & BHF-UCL & PMID:18505915 \\
\hline SMAD4 & Mothers against decapentaplegic homolog 4 & BHF-UCL & GO_REF:0000024 \\
\hline SMAD7 & Mothers against decapentaplegic homolog 7 & BHF-UCL & PMID:18762808 \\
\hline SNAI1 & Zinc finger protein SNAI1 & BHF-UCL & PMID:17072303 \\
\hline STRAP & Serine-threonine kinase receptor-associated protein & UniProt & PMID:23687415 \\
\hline TBX5 & T-box transcription factor TBX5 & DFLAT & PMID:20299672 \\
\hline TCF7L2 & Transcription factor 7-like 2 & BHF-UCL & PMID:17072303 \\
\hline TGFB1 & Transforming growth factor beta- 1 & BHF-UCL & PMID:21319131 \\
\hline
\end{tabular}




\begin{tabular}{|l|l|l|}
\hline TGFB1I1 & Transforming growth factor beta-1-induced transcript 1 protein & BHF-UCL \\
\hline TGFB2 & Transforming growth factor beta-2 & BHF-UCL \\
\hline TGFB3 & Transforming growth factor beta-3 & BHF-UCL \\
\hline TGFBR1 & Receptor protein serine/threonine kinase & AgBase \\
\hline TGFBR2 & TGF-beta receptor type-2 & BHF-UCL \\
\hline TRIM62 & E3 ubiquitin-protein ligase TRIM62 & Ensembl \\
\hline TWIST1 & Twist-related protein 1 & BHF-UCL \\
\hline URS0000170CF4_9606 & Homo sapiens (human) hsa-miR-221-3p. & BHF-UCL \\
\hline URS000039ED8D_9606 & Homo sapiens (human) hsa-miR-21-5p. & BHF-UCL \\
\hline VASN & Vasorin & UniProt \\
\hline WWTR1 & WW domain-containing transcription regulator protein 1 & PMID:26459119 \\
\hline ZNF703 & Zinc finger protein 703 & UniProt \\
\hline
\end{tabular}

A

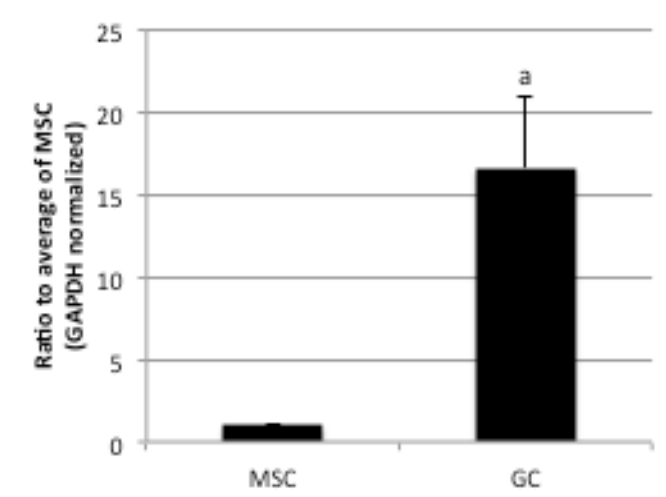

B

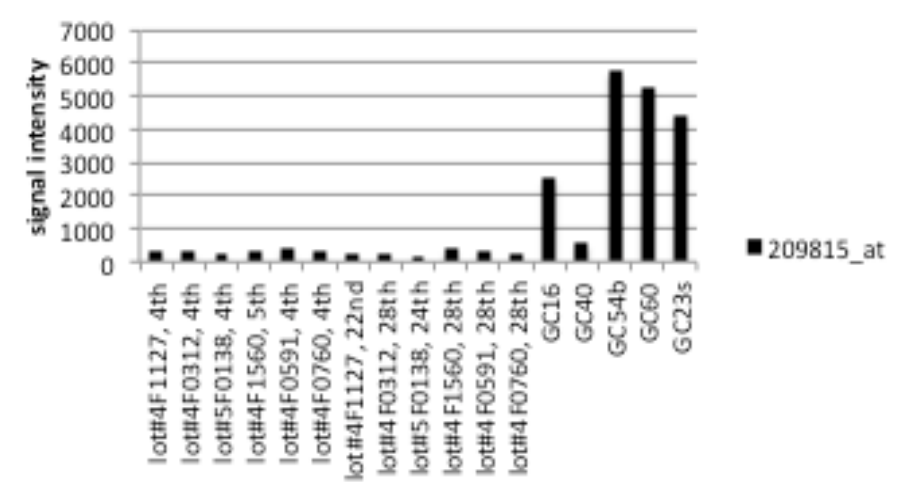

Figure 1. Gene expression of PTCH1 in MSCs and diffuse-type GC

A: Gene expression of PTCH1 was up-regulated in diffuse-type GC compared to MSCs ( ${ }^{\mathrm{a}} p<0.001$ in Student's $t$-test, $\mathrm{n}=12$ in MSCs, $\mathrm{n}=5$ in diffuse-type GC). The ratio to the average of microarray signal intensity in MSCs was calculated. B: Signal intensity in each sample of MSCs and diffuse-type GC are shown.

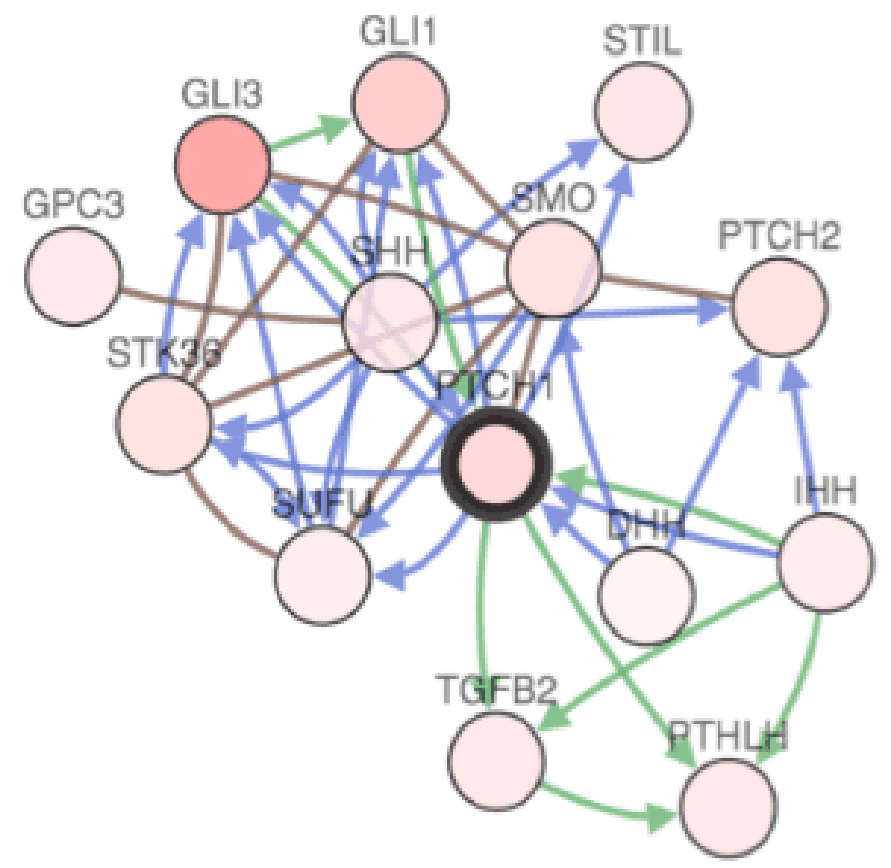

Figure 2. PTCH1 network in stomach adenocarcinoma (cBioPortal for Cancer Genomics) A PTCH1 model network was generated for stomach adenocarcinoma with cBioPortal for Cancer Genomics with query of PTCH1. extracted to generate the model network for PTCH1 and EMT-related genes, as shown in figure 3. In figure 3, the up-regulated (UR) genes in diffuse-type GC compared to MSCs are shown in red, whereas downregulated (DR) genes are shown in light purple (Fold change $(\mathrm{FC})>3$, $p<0.01)$. The genes in which FC of gene expression is between 2 and 3 and $\mathrm{p}$ value is less than 0.01 are highlighted with beige. UR genes in gastric cancer in the NextBio database are shown in an orange gradient, whereas DR genes are shown in green gradient. The genes in which expression was not altered in diffuse-type GC and MSCs are shown in light blue. A total of 60 genes were used to generate the PTCH1 model network.

In the model network generated from the pathway analysis shown in figure 3, notch 1 (NOTCH1), CTNNB1, stratifin (SFN), AKT serine/ threonine kinase 2 (AKT2), secreted frizzled related protein 2 (SFRP2), and claudin 1 (CLDN1) were UR in diffuse-type GC compared to MSCs. F11 receptor (F11R) and the Jun proto-oncogene AP-1 transcription factor subunit (JUN) were up-regulated in diffuse-type GC compared to MSCs, although these genes are shown as DR in gastric cancer based on the NextBio database. The gene expression of integrin subunit alpha 5 (ITGA5) was DR in diffuse-type GC compared to MSCs, although it is shown to be UR in gastric cancer based on NextBio database. We have previously shown that the gene expression of cadherin 1 (CDH1) was $\mathrm{UR}$, and $\mathrm{CDH} 2$ and fibronectin 1 (FN1) were DR in diffuse-type GC compared to MSCs [11]. Gene expression of Janus kinase 1 (JAK1), a signal transducer and activator of transcription 1 (STAT1) and STAT2 in the JAK-STAT pathway, was not altered in diffuse-type GC and 
Table 2. Genes queried for network analysis of cBioPortal for Cancer Genomics and IPA

\begin{tabular}{|c|c|}
\hline $\begin{array}{l}\text { Gene } \\
\text { symbol }\end{array}$ & Full name \\
\hline AKT1 & v-akt murine thymoma viral oncogene homolog 1 \\
\hline AKT2 & v-akt murine thymoma viral oncogene homolog 2 \\
\hline AKT3 & $\begin{array}{l}\text { v-akt murine thymoma viral oncogene homolog } 3 \text { (protein kinase B, } \\
\text { gamma) }\end{array}$ \\
\hline ALCAM & activated leukocyte cell adhesion molecule \\
\hline AXIN2 & $\operatorname{axin} 2$ \\
\hline BAMBI & BMP and activin membrane bound inhibitor \\
\hline BMI1 & BMI1 proto-oncogene, polycomb ring finger \\
\hline BMP2 & bone morphogenetic protein 2 \\
\hline CDH1 & cadherin 1 \\
\hline $\mathrm{CDH} 2$ & cadherin 2 \\
\hline CLDN1 & claudin 1 \\
\hline COL1A1 & collagen, type I, alpha 1 \\
\hline CTNNB1 & catenin (cadherin-associated protein), beta $1,88 \mathrm{kDa}$ \\
\hline CXADR & coxsackie virus and adenovirus receptor \\
\hline DAB2 & Dab, mitogen-responsive phosphoprotein, homolog 2 (Drosophila) \\
\hline EFNA1 & ephrin-A1 \\
\hline ELL3 & elongation factor RNA polymerase II-like 3 \\
\hline EPB41L5 & erythrocyte membrane protein band 4.1 like 5 \\
\hline EPCAM & epithelial cell adhesion molecule \\
\hline ЕРНА3 & EPH receptor A3 \\
\hline EZH2 & enhancer of zeste homolog 2 (Drosophila) \\
\hline F11R & F11 receptor \\
\hline FAM101B & family with sequence similarity 101 , member B \\
\hline FLNA & filamin A, alpha \\
\hline FN1 & fibronectin 1 \\
\hline FOXF2 & forkhead box F2 \\
\hline GJB2 & gap junction protein, beta $2,26 \mathrm{kDa}$ \\
\hline GREM1 & gremlin 1, DAN family BMP antagonist \\
\hline GSK3B & glycogen synthase kinase 3 beta \\
\hline HEY1 & hairy/enhancer-of-split related with YRPW motif 1 \\
\hline HEYL & hairy/enhancer-of-split related with YRPW motif-like \\
\hline HGF & hepatocyte growth factor (hepapoietin A; scatter factor) \\
\hline HIF1A & $\begin{array}{l}\text { hypoxia inducible factor 1, alpha subunit (basic helix-loop-helix } \\
\text { transcription factor) }\end{array}$ \\
\hline HMGA2 & high mobility group AT-hook 2 \\
\hline HNRNPAB & heterogeneous nuclear ribonucleoprotein $\mathrm{A} / \mathrm{B}$ \\
\hline ICAM3 & intercellular adhesion molecule 3 \\
\hline ITGA5 & integrin, alpha 5 (fibronectin receptor, alpha polypeptide) \\
\hline JAK1 & Janus kinase 1 \\
\hline JUN & jun proto-oncogene \\
\hline KRT8 & keratin 8 \\
\hline LEF1 & lymphoid enhancer-binding factor 1 \\
\hline LOXL2 & lysyl oxidase-like 2 \\
\hline LOXL3 & lysyl oxidase-like 3 \\
\hline MAD2L2 & MAD2 mitotic arrest deficient-like 2 (yeast) \\
\hline NANOG & Nanog homeobox \\
\hline NCAM1 & neural cell adhesion molecule 1 \\
\hline NFATC1 & nuclear factor of activated T-cells, cytoplasmic, calcineurin-dependent 1 \\
\hline NOTCH1 & notch 1 \\
\hline OCLN & occludin \\
\hline PDCD1 & programmed cell death 1 \\
\hline PECAM1 & platelet/endothelial cell adhesion molecule 1 \\
\hline PINK1 & PTEN induced putative kinase 1 \\
\hline POFUT2 & protein O-fucosyltransferase 2 \\
\hline PPP2CA & protein phosphatase 2 , catalytic subunit, alpha isozyme \\
\hline PRRX1 & paired related homeobox 1 \\
\hline PTCH1 & patched 1 \\
\hline RBPJ & recombination signal binding protein for immunoglobulin kappa $\mathrm{J}$ re \\
\hline
\end{tabular}

\begin{tabular}{|l|l|}
\hline RGCC & regulator of cell cycle \\
\hline RTN4 & reticulon 4 \\
\hline S100A4 & S100 calcium binding protein A4 \\
\hline SFN & stratifin \\
\hline SFRP1 & secreted frizzled-related protein 1 \\
\hline SFRP2 & secreted frizzled-related protein 2 \\
\hline SMAD2 & SMAD family member 2 \\
\hline SMAD3 & SMAD family member 3 \\
\hline SMAD4 & SMAD family member 4 \\
\hline SMAD7 & SMAD family member 7 \\
\hline SNAI2 & snail family zinc finger 2 \\
\hline SOX2 & SRY (sex determining region Y)-box 2 \\
\hline SOX9 & SRY (sex determining region Y)-box 9 \\
\hline SP1 & Sp1 transcription factor \\
\hline STAT1 & signal transducer and activator of transcription 1, 91 $\mathrm{kDa}$ \\
\hline STAT2 & signal transducer and activator of transcription 2, 113kDa \\
\hline TCF7L2 & transcription factor 7-like 2 (T-cell specific, HMG-box) \\
\hline TGFB1 & transforming growth factor, beta 1 \\
\hline TGFB1I1 & transforming growth factor beta 1 induced transcript 1 \\
\hline TGFB2 & transforming growth factor, beta 2 \\
\hline TGFB3 & transforming growth factor, beta 3 \\
\hline TGFBR1 & transforming growth factor, beta receptor 1 \\
\hline TGFBR3 & transforming growth factor, beta receptor 3 \\
\hline TJP1 & tight junction protein 1 \\
\hline TRIM28 & tripartite motif containing 28 \\
\hline TWIST1 & twist basic helix-loop-helix transcription factor 1 \\
\hline VIM & vimentin \\
\hline WNT5A & wingless-type MMTV integration site family, member 5A \\
\hline WWTR1 & WW domain containing transcription regulator 1 \\
\hline ZEB1 & zinc finger E-box binding homeobox 1 \\
\hline ZEB2 & zinc finger E-box binding homeobox 2 \\
\hline ZNF703 & zinc finger protein 703 \\
\hline
\end{tabular}

MSCs. BMI1 proto-oncogene, polycomb ring finger (BMI1), CDH2, high mobility group AT-hook 2 (HMGA2), neural cell adhesion molecule 1 (NCAM1) and VIM were included in network generated with cBioPortal for Cancer Genomics, which resulted in generation of PTCH1 model network without these genes due to the linkage was through neighbor genes not with query genes.

\section{Network generated with EMT-related genes in Ingenuity Pathway Analysis (IPA)}

The 89 genes including PTCH1 and EMT-related genes were used for generating networks in IPA (Table 2). The networks generate with IPA included 11 networks related to cellular movement or cellular development. The most significant network is shown in figure 4 . The molecules in the network includes activated leukocyte cell adhesion molecule (ALCAM), BMP and activin membrane bound inhibitor (BAMBI), CDH2, collagen type I alpha 1 chain (COL1A1), ephrin A1 (EFNA1), HMGA2, ITGA5, mitotic arrest deficient 2 like 2 (MAD2L2), platelet and endothelial cell adhesion molecule 1 (PECAM1), SFRP2, SMAD family member 4 (SMAD4), SMAD7, SNAI2, transforming growth factor beta receptor 1 (TGFBR1), TGFBR3, TWIST1, WW domain containing transcription regulator 1 (WWTR1). The function of the network was associated with cardiovascular system development and function, and cellular movement.

\section{PTCH1 interactors identified by BioGRID}

Table 3 shows the gene ontology of 88 interactors with PTCH1 analysed with the BioGRID database [16]. PTCH1 had 94 total unique 
Table 3. Gene Ontology (GO) analysis of 88 interactors of PTCH1 (BioGRID)

\begin{tabular}{|c|c|c|}
\hline Gene Symbol & Gene Title & GO biological process term \\
\hline ABCA2 & $\begin{array}{l}\text { ATP-binding cassette, sub-family A } \\
(\mathrm{ABC} 1) \text {, member } 2\end{array}$ & $\begin{array}{l}\text { ATP catabolic process / regulation of transcription from RNA polymerase II promoter / lipid metabolic process / transport / } \\
\text { metabolic process / regulation of intracellular cholesterol transport / response to drug / cholesterol homeostasis / response to } \\
\text { steroid hormone / transmembrane transport }\end{array}$ \\
\hline ADAM17 & ADAM metallopeptidase domain 17 & $\begin{array}{l}\text { response to hypoxia / positive regulation of protein phosphorylation / neutrophil mediated immunity / germinal center formation } \\
\text { / positive regulation of leukocyte chemotaxis / proteolysis / membrane protein ectodomain proteolysis / apoptotic process / } \\
\text { cell adhesion / epidermal growth factor receptor signaling pathway / Notch signaling pathway / Notch receptor processing / } \\
\text { positive regulation of cell proliferation / positive regulation of T cell chemotaxis / extracellular matrix disassembly / B cell } \\
\text { differentiation / extracellular matrix organization / positive regulation of cell growth / positive regulation of cell migration / } \\
\text { positive regulation of transforming growth factor beta receptor signaling pathway / negative regulation of transforming growth } \\
\text { factor beta receptor signaling pathway / collagen catabolic process / membrane protein intracellular domain proteolysis / } \\
\text { positive regulation of cyclin-dependent protein serine/threonine kinase activity involved in G/S transition of mitotic cell } \\
\text { cycle / response to lipopolysaccharide / negative regulation of interleukin-8 production / positive regulation of chemokine } \\
\text { production / regulation of mast cell apoptotic process / T cell differentiation in thymus / cell adhesion mediated by integrin / } \\
\text { wound healing, spreading of epidermal cells / epidermal growth factor-activated receptor transactivation by G-protein coupled } \\
\text { receptor signaling pathway / response to drug / positive regulation of epidermal growth factor-activated receptor activity } \\
\text { / neurotrophin TRK receptor signaling pathway / spleen development / cell motility / PMA-inducible membrane protein } \\
\text { ectodomain proteolysis / positive regulation of cellular component movement / response to high density lipoprotein particle / } \\
\text { JAK-STAT cascade involved in growth hormone signaling pathway / apoptotic signaling pathway }\end{array}$ \\
\hline ARSK & arylsulfatase family, member K & $\begin{array}{l}\text { sphingolipid metabolic process / glycosphingolipid metabolic process / metabolic process / post-translational protein } \\
\text { modification / cellular protein metabolic process / small molecule metabolic process }\end{array}$ \\
\hline ASPH & aspartate beta-hydroxylase & $\begin{array}{l}\text { detection of calcium ion / muscle contraction / pattern specification process / negative regulation of cell proliferation / positive } \\
\text { regulation of calcium ion transport into cytosol / regulation of cell communication by electrical coupling / regulation of release } \\
\text { of sequestered calcium ion into cytosol by sarcoplasmic reticulum / regulation of cardiac muscle contraction by regulation of } \\
\text { the release of sequestered calcium ion / peptidyl-amino acid modification / regulation of inositol 1,4,5-trisphosphate-sensitive } \\
\text { calcium-release channel activity / regulation of protein stability / activation of store-operated calcium channel activity / } \\
\text { response to ATP / limb morphogenesis / peptidyl-aspartic acid hydroxylation / positive regulation of proteolysis / positive } \\
\text { regulation of transcription, DNA-templated / oxidation-reduction process / palate development / regulation of ryanodine- } \\
\text { sensitive calcium-release channel activity / face morphogenesis / calcium ion transmembrane transport / cellular response } \\
\text { to calcium ion / positive regulation of intracellular protein transport / activation of cysteine-type endopeptidase activity / } \\
\text { regulation of protein depolymerization }\end{array}$ \\
\hline ATF6B & activating transcription factor 6 beta & transcription, DNA-templated / regulation of transcription, DNA-templated / response to unfolded protein / signal transduction \\
\hline B3GALTL & beta 1,3-galactosyltransferase-like & carbohydrate metabolic process / fucose metabolic process / protein glycosylation \\
\hline B4GALT1 & \begin{tabular}{|l|} 
UDP-Gal:betaGlcNAc beta 1,4- \\
galactosyltransferase, polypeptide 1
\end{tabular} \mid & $\begin{array}{l}\text { epithelial cell development / acute inflammatory response / carbohydrate metabolic process / lactose biosynthetic process / } \\
\text { galactose metabolic process / protein glycosylation / protein N-linked glycosylation / cell adhesion / Notch signaling pathway / } \\
\text { single fertilization / binding of sperm to zona pellucida / penetration of zona pellucida / negative regulation of cell proliferation } \\
\text { / glycoprotein biosynthetic process / oligosaccharide biosynthetic process / keratan sulfate biosynthetic process / protein } \\
\text { N-linked glycosylation via asparagine / extracellular matrix organization / glycosaminoglycan metabolic process / mammary } \\
\text { gland development / multicellular organism reproduction / wound healing / regulation of cell proliferation / keratan sulfate } \\
\text { metabolic process / positive regulation of apoptotic process / post-translational protein modification / cellular protein metabolic } \\
\text { process / small molecule metabolic process / development of secondary sexual characteristics / branching morphogenesis } \\
\text { of an epithelial tube / leukocyte migration / regulation of cellular component movement / regulation of acrosome reaction } \\
\text { / positive regulation of epithelial cell proliferation involved in wound healing / angiogenesis involved in wound healing / } \\
\text { positive regulation of apoptotic process involved in mammary gland involution }\end{array}$ \\
\hline B4GALT3 & $\begin{array}{l}\text { UDP-Gal:betaGlcNAc beta 1,4- } \\
\text { galactosyltransferase, polypeptide } 3\end{array}$ & $\begin{array}{l}\text { carbohydrate metabolic process / protein glycosylation / keratan sulfate biosynthetic process / protein N-linked glycosylation } \\
\text { via asparagine / glycosaminoglycan metabolic process / keratan sulfate metabolic process / post-translational protein } \\
\text { modification / cellular protein metabolic process / small molecule metabolic process }\end{array}$ \\
\hline B4GALT7 & $\begin{array}{l}\text { xylosylprotein beta } \\
1,4 \text {-galactosyltransferase, } \\
\text { polypeptide } 7\end{array}$ & $\begin{array}{l}\text { carbohydrate metabolic process / glycosaminoglycan biosynthetic process / proteoglycan metabolic process / cellular protein } \\
\text { modification process / protein glycosylation / protein N-linked glycosylation / glycosaminoglycan metabolic process / } \\
\text { chondroitin sulfate metabolic process / extracellular fibril organization / small molecule metabolic process / negative regulation } \\
\text { of fibroblast proliferation }\end{array}$ \\
\hline CACNA2D1 & $\begin{array}{l}\text { calcium channel, voltage-dependent, } \\
\text { alpha } 2 \text { /delta subunit } 1\end{array}$ & $\begin{array}{l}\text { transport / ion transport / calcium ion transport / regulation of ion transmembrane transport / regulation of calcium ion transport } \\
\text { / calcium ion transmembrane transport }\end{array}$ \\
\hline CACNA2D2 & $\begin{array}{l}\text { calcium channel, voltage-dependent, } \\
\text { alpha } 2 / \text { delta subunit } 2\end{array}$ & $\begin{array}{l}\text { energy reserve metabolic process / transport / ion transport / calcium ion transport / neuromuscular junction development / } \\
\text { regulation of ion transmembrane transport / regulation of multicellular organism growth / small molecule metabolic process / } \\
\text { positive regulation of organ growth / muscle fiber development/regulation of insulin secretion / rhythmic synaptic transmission } \\
\text { / calcium ion transmembrane transport }\end{array}$ \\
\hline CASP9 & \begin{tabular}{|l|} 
\\
caspase 9, apoptosis-related cysteine \\
peptidase
\end{tabular} & $\begin{array}{l}\text { proteolysis / apoptotic process / activation of cysteine-type endopeptidase activity involved in apoptotic process / cellular } \\
\text { response to DNA damage stimulus / epidermal growth factor receptor signaling pathway / aging / fibroblast growth factor } \\
\text { receptor signaling pathway / intrinsic apoptotic signaling pathway in response to DNA damage / activation of cysteine-type } \\
\text { endopeptidase activity involved in apoptotic process by cytochrome c / response to UV / response to organic cyclic compound } \\
\text { / platelet formation / response to cobalt ion / response to estradiol / response to lipopolysaccharide / glial cell apoptotic process } \\
\text { / cellular response to UV / Fc-epsilon receptor signaling pathway / signal transduction in response to DNA damage / regulation } \\
\text { of apoptotic process / positive regulation of apoptotic process / positive regulation of neuron apoptotic process / innate immune } \\
\text { response / response to antibiotic / neurotrophin TRK receptor signaling pathway / phosphatidylinositol-mediated signaling } \\
\text { / cellular response to organic cyclic compound / cellular response to dexamethasone stimulus / intrinsic apoptotic signaling } \\
\text { pathway / regulation of response to DNA damage stimulus }\end{array}$ \\
\hline
\end{tabular}




\begin{tabular}{|c|c|c|}
\hline CAV1 & caveolin 1, caveolae protein, $22 \mathrm{kDa}$ & 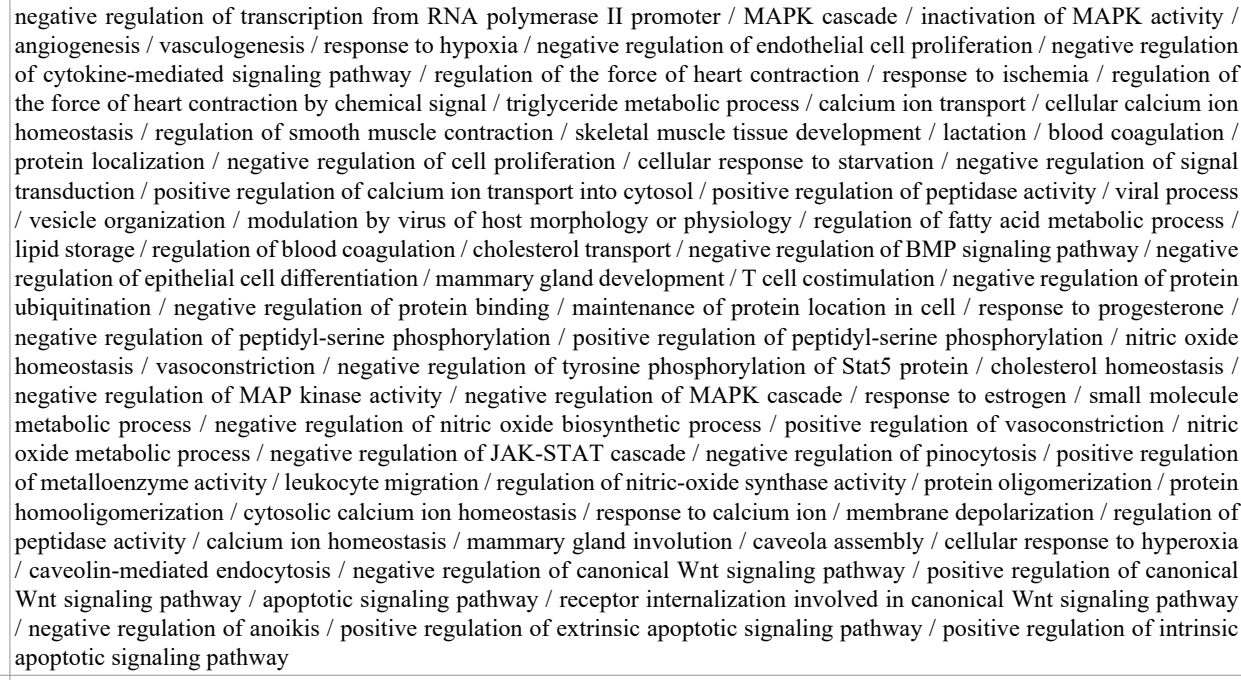 \\
\hline CCPG1 & cell cycle progression 1 & $\begin{array}{l}\text { cell cycle / positive regulation of cell proliferation / positive regulation of cell cycle / positive regulation of transcription from } \\
\text { RNA polymerase II promoter / regulation of Rho guanyl-nucleotide exchange factor activity }\end{array}$ \\
\hline CHPF & chondroitin polymerizing factor & $\begin{array}{l}\text { carbohydrate metabolic process / metabolic process / glycosaminoglycan metabolic process / chondroitin sulfate metabolic } \\
\text { process / chondroitin sulfate biosynthetic process / small molecule metabolic process }\end{array}$ \\
\hline CHST12 & $\begin{array}{l}\text { carbohydrate (chondroitin 4) } \\
\text { sulfotransferase } 12\end{array}$ & $\begin{array}{l}\text { carbohydrate metabolic process / carbohydrate biosynthetic process / glycosaminoglycan metabolic process / chondroitin } \\
\text { sulfate metabolic process / chondroitin sulfate biosynthetic process / dermatan sulfate biosynthetic process / small molecule } \\
\text { metabolic process }\end{array}$ \\
\hline CHST14 & $\begin{array}{l}\text { carbohydrate } \\
\text { (N-acetylgalactosamine 4-0) } \\
\text { sulfotransferase } 14\end{array}$ & $\begin{array}{l}\text { carbohydrate metabolic process / carbohydrate biosynthetic process / glycosaminoglycan metabolic process / chondroitin } \\
\text { sulfate metabolic process / dermatan sulfate biosynthetic process / small molecule metabolic process / dermatan sulfate } \\
\text { proteoglycan metabolic process }\end{array}$ \\
\hline CHSY1 & chondroitin sulfate synthase 1 & $\begin{array}{l}\text { carbohydrate metabolic process / metabolic process / glycosaminoglycan metabolic process / chondroitin sulfate metabolic } \\
\text { process / chondroitin sulfate biosynthetic process / negative regulation of ossification / response to nutrient levels / small } \\
\text { molecule metabolic process }\end{array}$ \\
\hline CNNM1 & cyclin M1 & transport / ion transport \\
\hline CNNM4 & cyclin M4 & transport / ion transport / visual perception / biomineral tissue development / response to stimulus \\
\hline CNTNAP3 & contactin associated protein-like 3 & cell adhesion / cell recognition \\
\hline $\mathrm{COCH}$ & cochlin & sensory perception of sound / regulation of cell shape \\
\hline COL18A1 & collagen, type XVIII, alpha 1 & $\begin{array}{l}\text { angiogenesis / endothelial cell morphogenesis / cell adhesion / visual perception / positive regulation of cell proliferation } \\
\text { / negative regulation of cell proliferation / organ morphogenesis / extracellular matrix disassembly / extracellular matrix } \\
\text { organization / positive regulation of cell migration / collagen catabolic process / response to drug / positive regulation of } \\
\text { apoptotic process / response to hydrostatic pressure / positive regulation of endothelial cell apoptotic process }\end{array}$ \\
\hline DDX19B & $\begin{array}{l}\text { DEAD (Asp-Glu-Ala-Asp) box } \\
\text { polypeptide 19B }\end{array}$ & mRNA export from nucleus / transport / metabolic process / protein transport / mRNA transport \\
\hline DHFRL1 & dihydrofolate reductase-like 1 & $\begin{array}{l}\text { glycine biosynthetic process / one-carbon metabolic process / nucleotide biosynthetic process / thymidine biosynthetic process } \\
\text { / tetrahydrofolate metabolic process / tetrahydrofolate biosynthetic process / oxidation-reduction process }\end{array}$ \\
\hline EGFR & epidermal growth factor receptor & 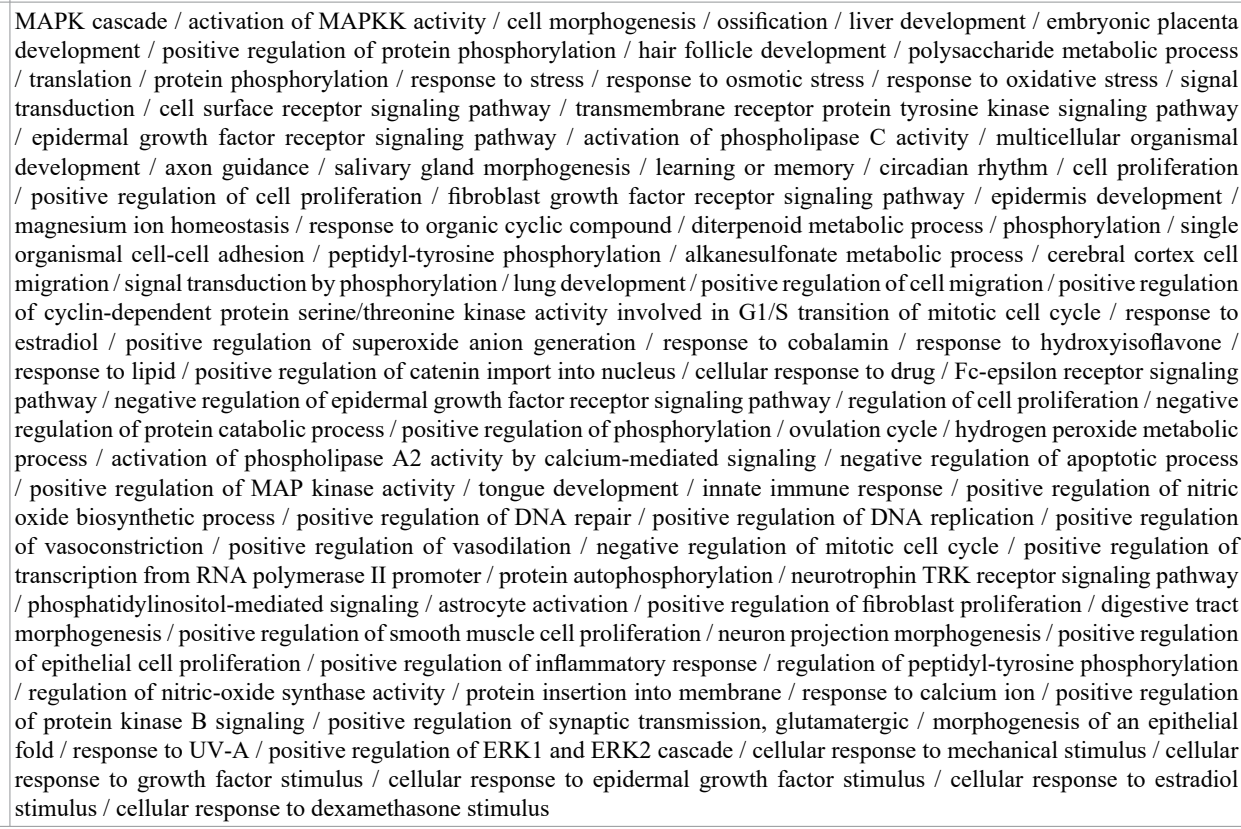 \\
\hline
\end{tabular}




\begin{tabular}{|c|c|c|}
\hline ENDOG & endonuclease G & $\begin{array}{l}\text { in utero embryonic development / apoptotic DNA fragmentation / DNA recombination / response to tumor necrosis factor / } \\
\text { positive regulation of apoptotic process / response to antibiotic }\end{array}$ \\
\hline ENPP1 & $\begin{array}{l}\text { ectonucleotide pyrophosphatase/ } \\
\text { phosphodiesterase } 1\end{array}$ & $\begin{array}{l}\text { generation of precursor metabolites and energy / ATP catabolic process / vitamin metabolic process / water-soluble vitamin } \\
\text { metabolic process / riboflavin metabolic process / phosphate-containing compound metabolic process / receptor-mediated } \\
\text { endocytosis / immune response / metabolic process / nucleoside triphosphate catabolic process / negative regulation of } \\
\text { ossification / negative regulation of cell growth / regulation of bone mineralization / inorganic diphosphate transport / cellular } \\
\text { phosphate ion homeostasis / sequestering of triglyceride / biomineral tissue development / negative regulation of protein } \\
\text { autophosphorylation / cellular response to insulin stimulus / small molecule metabolic process / negative regulation of fat } \\
\text { cell differentiation / negative regulation of glycogen biosynthetic process / negative regulation of glucose import / negative } \\
\text { regulation of insulin receptor signaling pathway / bone remodeling / 3'-phosphoadenosine 5'-phosphosulfate metabolic process } \\
\text { / nucleic acid phosphodiester bond hydrolysis }\end{array}$ \\
\hline EXT1 & exostosin glycosyltransferase 1 & $\begin{array}{l}\text { skeletal system development / ossification / carbohydrate metabolic process / glycosaminoglycan biosynthetic process / protein } \\
\text { glycosylation / signal transduction / gastrulation / axon guidance / brain development / endoderm development / mesoderm } \\
\text { development / heparan sulfate proteoglycan biosynthetic process / polysaccharide chain biosynthetic process / olfactory bulb } \\
\text { development / glycosaminoglycan metabolic process / cellular polysaccharide biosynthetic process / small molecule metabolic } \\
\text { process / embryonic skeletal joint development }\end{array}$ \\
\hline FHL2 & four and a half LIM domains 2 & $\begin{array}{l}\text { negative regulation of transcription from RNA polymerase II promoter / osteoblast differentiation / transcription, DNA- } \\
\text { templated / regulation of transcription, DNA-templated / regulation of transcription from RNA polymerase II promoter / } \\
\text { response to hormone / androgen receptor signaling pathway / negative regulation of apoptotic process / cellular lipid metabolic } \\
\text { process / small molecule metabolic process / negative regulation of transcription, DNA-templated / positive regulation of } \\
\text { transcription, DNA-templated / atrial cardiac muscle cell development / ventricular cardiac muscle cell development / heart } \\
\text { trabecula formation }\end{array}$ \\
\hline FUT11 & $\begin{array}{l}\text { fucosyltransferase } 11 \text { (alpha }(1,3) \\
\text { fucosyltransferase) }\end{array}$ & protein glycosylation / fucosylation \\
\hline GALNT7 & $\begin{array}{l}\text { polypeptide } \\
\text { N-acetylgalactosaminyltransferase } 7\end{array}$ & $\begin{array}{l}\text { carbohydrate metabolic process / protein glycosylation / protein O-linked glycosylation / metabolic process / O-glycan } \\
\text { processing / post-translational protein modification / cellular protein metabolic process }\end{array}$ \\
\hline GCNT1 & $\begin{array}{l}\text { glucosaminyl (N-acetyl) transferase } \\
1, \text { core } 2\end{array}$ & $\begin{array}{l}\text { protein glycosylation / metabolic process / glycoprotein biosynthetic process / O-glycan processing / response to insulin / post- } \\
\text { translational protein modification / cellular protein metabolic process / tissue morphogenesis / leukocyte tethering or rolling / } \\
\text { cell adhesion molecule production / kidney morphogenesis }\end{array}$ \\
\hline HLA-A & $\begin{array}{l}\text { major histocompatibility complex, } \\
\text { class I, A }\end{array}$ & $\begin{array}{l}\text { positive regulation of T cell mediated cytotoxicity / immune system process / antigen processing and presentation of peptide } \\
\text { antigen via MHC class I / antigen processing and presentation of exogenous peptide antigen via MHC class I, TAP-dependent } \\
\text { / antigen processing and presentation of exogenous peptide antigen via MHC class I, TAP-independent / antigen processing } \\
\text { and presentation of endogenous peptide antigen via MHC class I via ER pathway, TAP-independent / immune response / } \\
\text { viral process / detection of bacterium / cytokine-mediated signaling pathway / antigen processing and presentation / positive } \\
\text { regulation of interferon-gamma production / protection from natural killer cell mediated cytotoxicity / antigen processing and } \\
\text { presentation of exogenous peptide antigen via MHC class I / regulation of defense response to virus by virus / regulation of } \\
\text { immune response / interferon-gamma-mediated signaling pathway / type I interferon signaling pathway / positive regulation } \\
\text { of memory T cell activation }\end{array}$ \\
\hline HLA-C & $\begin{array}{l}\text { major histocompatibility complex, } \\
\text { class I, C }\end{array}$ & $\begin{array}{l}\text { cilium or flagellum-dependent cell motility / positive regulation of T cell mediated cytotoxicity / immune system process / } \\
\text { antigen processing and presentation of peptide antigen via MHC class I / antigen processing and presentation of exogenous } \\
\text { peptide antigen via MHC class I, TAP-dependent / antigen processing and presentation of exogenous peptide antigen via } \\
\text { MHC class I, TAP-independent / antigen processing and presentation of endogenous peptide antigen via MHC class I via ER } \\
\text { pathway, TAP-independent / immune response / microtubule-based movement / signal transduction / metabolic process / viral } \\
\text { process / modulation by virus of host morphology or physiology / cytokine-mediated signaling pathway / antigen processing } \\
\text { and presentation / antigen processing and presentation of exogenous peptide antigen via MHC class I / innate immune response } \\
\text { / regulation of immune response / interferon-gamma-mediated signaling pathway / type I interferon signaling pathway }\end{array}$ \\
\hline HS6ST2 & $\begin{array}{l}\text { heparan sulfate 6-O-sulfotransferase } \\
2\end{array}$ & $\begin{array}{l}\text { carbohydrate metabolic process / glycosaminoglycan biosynthetic process / metabolic process / glycosaminoglycan metabolic } \\
\text { process / small molecule metabolic process }\end{array}$ \\
\hline HSPA5 & $\begin{array}{l}\text { heat shock } 70 \mathrm{kDa} \text { protein } 5 \\
\text { (glucose-regulated protein, } 78 \mathrm{kDa} \text { ) }\end{array}$ & $\begin{array}{l}\text { cell morphogenesis / platelet degranulation / ATP catabolic process / ER overload response / activation of signaling protein } \\
\text { activity involved in unfolded protein response / blood coagulation / cerebellum structural organization / cerebellar Purkinje cell } \\
\text { layer development / substantia nigra development / platelet activation / positive regulation of cell migration / ER-associated } \\
\text { ubiquitin-dependent protein catabolic process / negative regulation of transforming growth factor beta receptor signaling } \\
\text { pathway / endoplasmic reticulum unfolded protein response / positive regulation of protein ubiquitination / maintenance of } \\
\text { protein localization in endoplasmic reticulum / cellular response to glucose starvation / negative regulation of apoptotic process } \\
\text { / cellular protein metabolic process / proteolysis involved in cellular protein catabolic process / regulation of protein folding in } \\
\text { endoplasmic reticulum / cellular response to antibiotic / cellular response to interleukin-4 }\end{array}$ \\
\hline IMPAD1 & $\begin{array}{l}\text { inositol monophosphatase domain } \\
\text { containing } 1\end{array}$ & $\begin{array}{l}\text { skeletal system development / endochondral ossification / chondrocyte development / inositol biosynthetic process / post- } \\
\text { embryonic development / dephosphorylation / chondroitin sulfate metabolic process / embryonic digit morphogenesis / } \\
\text { phosphatidylinositol phosphorylation }\end{array}$ \\
\hline ITCH & itchy E3 ubiquitin protein ligase & $\begin{array}{l}\text { protein polyubiquitination / regulation of cell growth / immune system process / positive regulation of T cell anergy / ubiquitin- } \\
\text { dependent protein catabolic process / apoptotic process / inflammatory response / Notch signaling pathway / metabolic process } \\
\text { / viral process / protein ubiquitination / modulation by virus of host morphology or physiology / negative regulation of NF- } \\
\text { kappaB transcription factor activity / negative regulation of type I interferon production / protein K29-linked ubiquitination } \\
\text { / nucleotide-binding domain, leucine rich repeat containing receptor signaling pathway / protein ubiquitination involved } \\
\text { in ubiquitin-dependent protein catabolic process / negative regulation of apoptotic process / innate immune response / } \\
\text { positive regulation of protein catabolic process / negative regulation of JNK cascade / negative regulation of alpha-beta T } \\
\text { cell proliferation / viral entry into host cell / negative regulation of defense response to virus / defense response to virus / } \\
\text { nucleotide-binding oligomerization domain containing signaling pathway / protein K63-linked ubiquitination / protein K48- } \\
\text { linked ubiquitination / regulation of protein deubiquitination }\end{array}$ \\
\hline ITGA4 & $\begin{array}{l}\text { integrin, alpha } 4 \text { (antigen CD49D, } \\
\text { alpha } 4 \text { subunit of VLA-4 receptor) }\end{array}$ & $\begin{array}{l}\text { blood vessel remodeling / cell adhesion / heterophilic cell-cell adhesion / leukocyte cell-cell adhesion / integrin-mediated } \\
\text { signaling pathway / heart development / blood coagulation / cell migration / B cell differentiation / extracellular matrix } \\
\text { organization / regulation of immune response / leukocyte migration / face development / chorio-allantoic fusion / negative } \\
\text { regulation of protein homodimerization activity }\end{array}$ \\
\hline
\end{tabular}




\begin{tabular}{|c|c|c|}
\hline ITGA6 & integrin, alpha 6 & $\begin{array}{l}\text { cell-substrate junction assembly / cell adhesion / cell-matrix adhesion / integrin-mediated signaling pathway / blood coagulation } \\
\text { / positive regulation of cell-substrate adhesion / single organismal cell-cell adhesion / positive regulation of cell-cell adhesion } \\
\text { / extracellular matrix organization / hemidesmosome assembly / cell-substrate adhesion / cellular response to extracellular } \\
\text { stimulus / cell adhesion mediated by integrin / cell junction assembly / positive regulation of phosphorylation / odontogenesis } \\
\text { of dentin-containing tooth / positive regulation of apoptotic process / positive regulation of transcription from RNA polymerase } \\
\text { II promoter / filopodium assembly / brown fat cell differentiation / leukocyte migration / cellular response to organic cyclic } \\
\text { compound / negative regulation of extrinsic apoptotic signaling pathway }\end{array}$ \\
\hline ITGA7 & integrin, alpha 7 & $\begin{array}{l}\text { cell adhesion / cell-matrix adhesion / integrin-mediated signaling pathway / muscle organ development / skeletal muscle tissue } \\
\text { development / regulation of cell shape / cell migration / extracellular matrix organization / blood vessel morphogenesis }\end{array}$ \\
\hline ITGA8 & integrin, alpha 8 & $\begin{array}{l}\text { metanephros development / cell adhesion / cell-matrix adhesion / integrin-mediated signaling pathway / multicellular } \\
\text { organismal development / nervous system development / brain development / memory / single organismal cell-cell adhesion / } \\
\text { cell projection organization / cell differentiation / extracellular matrix organization / positive regulation of transforming growth } \\
\text { factor beta receptor signaling pathway / inner ear morphogenesis / establishment of protein localization / smooth muscle } \\
\text { tissue development / positive regulation of transcription from RNA polymerase II promoter involved in smooth muscle cell } \\
\text { differentiation }\end{array}$ \\
\hline KIAA1161 & KIAA1161 & $\begin{array}{l}\text { carbohydrate metabolic process / metabolic process / positive regulation of insulin-like growth factor receptor signaling } \\
\text { pathway / skeletal muscle fiber development / positive regulation of protein kinase B signaling }\end{array}$ \\
\hline LRFN3 & $\begin{array}{l}\text { leucine rich repeat and fibronectin } \\
\text { type III domain containing } 3\end{array}$ & cell adhesion \\
\hline MAN2A1 & $\begin{array}{l}\text { mannosidase, alpha, class } 2 \mathrm{~A}, \\
\text { member } 1\end{array}$ & $\begin{array}{l}\text { in utero embryonic development / liver development / carbohydrate metabolic process / mannose metabolic process / protein } \\
\text { glycosylation / N-glycan processing / mitochondrion organization / vacuole organization / respiratory gaseous exchange / } \\
\text { metabolic process / protein N-linked glycosylation via asparagine / post-translational protein modification / cellular protein } \\
\text { metabolic process / lung alveolus development / positive regulation of neurogenesis / retina morphogenesis in camera-type eye }\end{array}$ \\
\hline MGAT1 & $\begin{array}{l}\text { mannosyl (alpha-1,3-)- } \\
\text { glycoprotein beta-1,2-N- } \\
\text { acetylglucosaminyltransferase }\end{array}$ & $\begin{array}{l}\text { in utero embryonic development / carbohydrate metabolic process / UDP-N-acetylglucosamine catabolic process / protein } \\
\text { glycosylation / protein N-linked glycosylation via asparagine / post-translational protein modification / cellular protein } \\
\text { metabolic process }\end{array}$ \\
\hline MGAT5 & $\begin{array}{l}\text { mannosyl (alpha-1,6-)- } \\
\text { glycoprotein beta-1,6-N-acetyl- } \\
\text { glucosaminyltransferase }\end{array}$ & $\begin{array}{l}\text { protein glycosylation / protein N-linked glycosylation / protein N-linked glycosylation via asparagine / post-translational } \\
\text { protein modification / cellular protein metabolic process }\end{array}$ \\
\hline MOXD1 & monooxygenase, DBH-like 1 & catecholamine metabolic process / oxidation-reduction process \\
\hline MUCL1 & mucin-like 1 & O-glycan processing / post-translational protein modification / cellular protein metabolic process \\
\hline NDST2 & $\begin{array}{l}\text { N-deacetylase/N-sulfotransferase } \\
\text { (heparan glucosaminyl) } 2\end{array}$ & $\begin{array}{l}\text { carbohydrate metabolic process / glycosaminoglycan biosynthetic process / metabolic process / heparan sulfate proteoglycan } \\
\text { biosynthetic process / glycosaminoglycan metabolic process / heparin biosynthetic process / small molecule metabolic process }\end{array}$ \\
\hline NEDD4L & $\begin{array}{l}\text { neural precursor cell expressed, } \\
\text { developmentally down-regulated } \\
\text { 4-like, E3 ubiquitin protein ligase }\end{array}$ & $\begin{array}{l}\text { negative regulation of transcription from RNA polymerase II promoter / negative regulation of systemic arterial blood pressure } \\
\text { / regulation of membrane depolarization / transcription, DNA-templated / transcription initiation from RNA polymerase II } \\
\text { promoter / protein monoubiquitination / sodium ion transport / cellular sodium ion homeostasis / transforming growth factor } \\
\text { beta receptor signaling pathway / excretion / metabolic process / response to salt stress / response to metal ion / gene expression } \\
\text { / positive regulation of sodium ion transport / negative regulation of sodium ion transport / viral process / protein ubiquitination } \\
\text { / modulation by virus of host morphology or physiology / viral life cycle / water homeostasis / negative regulation of } \\
\text { transforming growth factor beta receptor signaling pathway / ion transmembrane transport / regulation of ion transmembrane } \\
\text { transport / regulation of protein catabolic process / regulation of membrane potential / protein ubiquitination involved in } \\
\text { ubiquitin-dependent protein catabolic process / proteasome-mediated ubiquitin-dependent protein catabolic process / ubiquitin- } \\
\text { dependent protein catabolic process via the multivesicular body sorting pathway / positive regulation of protein catabolic } \\
\text { process / positive regulation of endocytosis / transmembrane transport / regulation of membrane repolarization / protein } \\
\text { K48-linked ubiquitination / ventricular cardiac muscle cell action potential / regulation of potassium ion transmembrane } \\
\text { transporter activity / negative regulation of potassium ion transmembrane transporter activity / negative regulation of potassium } \\
\text { ion transmembrane transport / negative regulation of sodium ion transmembrane transport / negative regulation of protein } \\
\text { localization to cell surface / negative regulation of sodium ion transmembrane transporter activity / regulation of tight junction } \\
\text { assembly / positive regulation of cation channel activity / positive regulation of caveolin-mediated endocytosis }\end{array}$ \\
\hline NEDD4 & $\begin{array}{l}\text { neural precursor cell expressed, } \\
\text { developmentally down-regulated } 4, \\
\text { E3 ubiquitin protein ligase }\end{array}$ & $\begin{array}{l}\text { negative regulation of transcription from RNA polymerase II promoter / adaptive immune response / outflow tract } \\
\text { morphogenesis / endocardial cushion development / protein monoubiquitination / protein targeting to lysosome / lysosomal } \\
\text { transport / nervous system development / neuromuscular junction development / metabolic process / negative regulation of } \\
\text { sodium ion transport / negative regulation of transcription from RNA polymerase II promoter in response to UV-induced DNA } \\
\text { damage / positive regulation of phosphatidylinositol 3-kinase signaling / viral process / protein ubiquitination / modulation by } \\
\text { virus of host morphology or physiology / transmission of virus / cytokine-mediated signaling pathway / negative regulation } \\
\text { of vascular endothelial growth factor receptor signaling pathway / neuron projection development / receptor internalization / } \\
\text { receptor catabolic process / cellular response to UV / regulation of ion transmembrane transport / T cell activation / regulation } \\
\text { of membrane potential / protein ubiquitination involved in ubiquitin-dependent protein catabolic process / glucocorticoid } \\
\text { receptor signaling pathway / ubiquitin-dependent protein catabolic process via the multivesicular body sorting pathway } \\
\text { / development involved in symbiotic interaction / positive regulation of protein catabolic process / positive regulation of } \\
\text { nucleocytoplasmic transport / blood vessel morphogenesis / regulation of dendrite morphogenesis / regulation of synapse } \\
\text { organization / progesterone receptor signaling pathway / response to calcium ion / protein K63-linked ubiquitination / } \\
\text { regulation of potassium ion transmembrane transporter activity }\end{array}$ \\
\hline NETO2 & $\begin{array}{l}\text { neuropilin (NRP) and tolloid (TLL)- } \\
\text { like } 2\end{array}$ & regulation of kainate selective glutamate receptor activity \\
\hline PAM & $\begin{array}{l}\text { peptidylglycine alpha-amidating } \\
\text { monooxygenase }\end{array}$ & $\begin{array}{l}\text { peptide amidation / response to hypoxia / long-chain fatty acid metabolic process / regulation of transcription from RNA } \\
\text { polymerase II promoter / cellular protein modification process / peptide metabolic process / mitotic chromosome condensation } \\
\text { / central nervous system development / heart development / lactation / metabolic process / response to } \mathrm{pH} / \text { toxin metabolic } \\
\text { process / protein amidation / protein metabolic process / ovulation cycle process / response to estradiol / regulation of actin } \\
\text { cytoskeleton organization / odontogenesis / response to drug / response to copper ion / regulation of protein secretion / } \\
\text { protein homooligomerization / response to glucocorticoid / oxidation-reduction process / maternal process involved in female } \\
\text { pregnancy / limb development }\end{array}$ \\
\hline
\end{tabular}




\begin{tabular}{|c|c|c|}
\hline PDF & peptide deformylase, mitochondrial & $\begin{array}{l}\text { co-translational protein modification / translation / positive regulation of cell proliferation / peptidyl-methionine modification } \\
\text { / N-terminal protein amino acid modification }\end{array}$ \\
\hline PLXNA1 & plexin A1 & $\begin{array}{l}\text { signal transduction / multicellular organismal development / axon guidance / regulation of smooth muscle cell migration / } \\
\text { positive regulation of GTPase activity / regulation of small GTPase mediated signal transduction / dichotomous subdivision of } \\
\text { terminal units involved in salivary gland branching / semaphorin-plexin signaling pathway }\end{array}$ \\
\hline PLXNB2 & plexin B2 & $\begin{array}{l}\text { neural tube closure / regulation of protein phosphorylation / signal transduction / multicellular organismal development / } \\
\text { neuroblast proliferation / brain development/regulation of cell shape / regulation of Rho GTPase activity / positive regulation of } \\
\text { GTPase activity / positive regulation of axonogenesis / regulation of small GTPase mediated signal transduction / semaphorin- } \\
\text { plexin signaling pathway / regulation of neuron migration }\end{array}$ \\
\hline POMGNT2 & $\begin{array}{l}\text { protein O-linked mannose } \\
\mathrm{N} \text {-acetylglucosaminyltransferase } 2 \\
\text { (beta 1,4-) }\end{array}$ & protein glycosylation / protein O-linked glycosylation \\
\hline POMT2 & protein-O-mannosyltransferase 2 & protein glycosylation / protein O-linked glycosylation / protein O-linked mannosylation / mannosylation \\
\hline PXYLP1 & 2-phosphoxylose phosphatase 1 & metabolic process / dephosphorylation \\
\hline RNF130 & ring finger protein 130 & apoptotic process / programmed cell death / protein ubiquitination \\
\hline SEMA4F & $\begin{array}{l}\text { sema domain, immunoglobulin } \\
\text { domain (Ig), transmembrane domain } \\
\text { (TM) and short cytoplasmic domain, } \\
\text { (semaphorin) } 4 \mathrm{~F}\end{array}$ & $\begin{array}{l}\text { cell-cell signaling / multicellular organismal development / nervous system development / axon guidance / cell differentiation } \\
\text { / negative regulation of axon extension / retinal ganglion cell axon guidance }\end{array}$ \\
\hline SEMA6A & $\begin{array}{l}\text { sema domain, transmembrane } \\
\text { domain (TM), and cytoplasmic } \\
\text { domain, (semaphorin) 6A }\end{array}$ & $\begin{array}{l}\text { neuron migration / apoptotic process / cytoskeleton organization / cell surface receptor signaling pathway / multicellular } \\
\text { organismal development / nervous system development / axon guidance / organ morphogenesis / cell differentiation / } \\
\text { centrosome localization / semaphorin-plexin signaling pathway / positive regulation of neuron migration }\end{array}$ \\
\hline SLIT2 & slit homolog 2 (Drosophila) & $\begin{array}{l}\text { metanephros development / ureteric bud development / in utero embryonic development / negative regulation of protein } \\
\text { phosphorylation / cell migration involved in sprouting angiogenesis / negative regulation of leukocyte chemotaxis / chemotaxis } \\
\text { / multicellular organismal development / nervous system development / axonogenesis / axon guidance / motor neuron axon } \\
\text { guidance / negative regulation of cell proliferation / negative regulation of lamellipodium assembly / negative regulation of } \\
\text { endothelial cell migration / negative regulation of gene expression / negative regulation of smooth muscle cell migration / single } \\
\text { organismal cell-cell adhesion / olfactory bulb development / chemorepulsion involved in embryonic olfactory bulb interneuron } \\
\text { precursor migration / chemorepulsion involved in postnatal olfactory bulb interneuron migration / corticospinal neuron axon } \\
\text { guidance through spinal cord / telencephalon cell migration / cell differentiation / negative regulation of cell growth / negative } \\
\text { regulation of cell migration / negative regulation of actin filament polymerization / retinal ganglion cell axon guidance / cellular } \\
\text { response to hormone stimulus / dorsal/ventral axon guidance / Roundabout signaling pathway / positive regulation of apoptotic } \\
\text { process / negative regulation of catalytic activity / negative regulation of vascular permeability / branching morphogenesis } \\
\text { of an epithelial tube / neuron projection morphogenesis / axon extension involved in axon guidance / positive regulation of } \\
\text { axonogenesis / negative chemotaxis / induction of negative chemotaxis / negative regulation of small GTPase mediated signal } \\
\text { transduction / response to cortisol / mammary gland duct morphogenesis / mammary duct terminal end bud growth / apoptotic } \\
\text { process involved in luteolysis / negative regulation of chemokine-mediated signaling pathway / cellular response to heparin / } \\
\text { negative regulation of smooth muscle cell chemotaxis / negative regulation of mononuclear cell migration / negative regulation } \\
\text { of neutrophil chemotaxis / negative regulation of monocyte chemotaxis / negative regulation of retinal ganglion cell axon } \\
\text { guidance / negative regulation of cellular response to growth factor stimulus }\end{array}$ \\
\hline SMO & smoothened, frizzled class receptor & 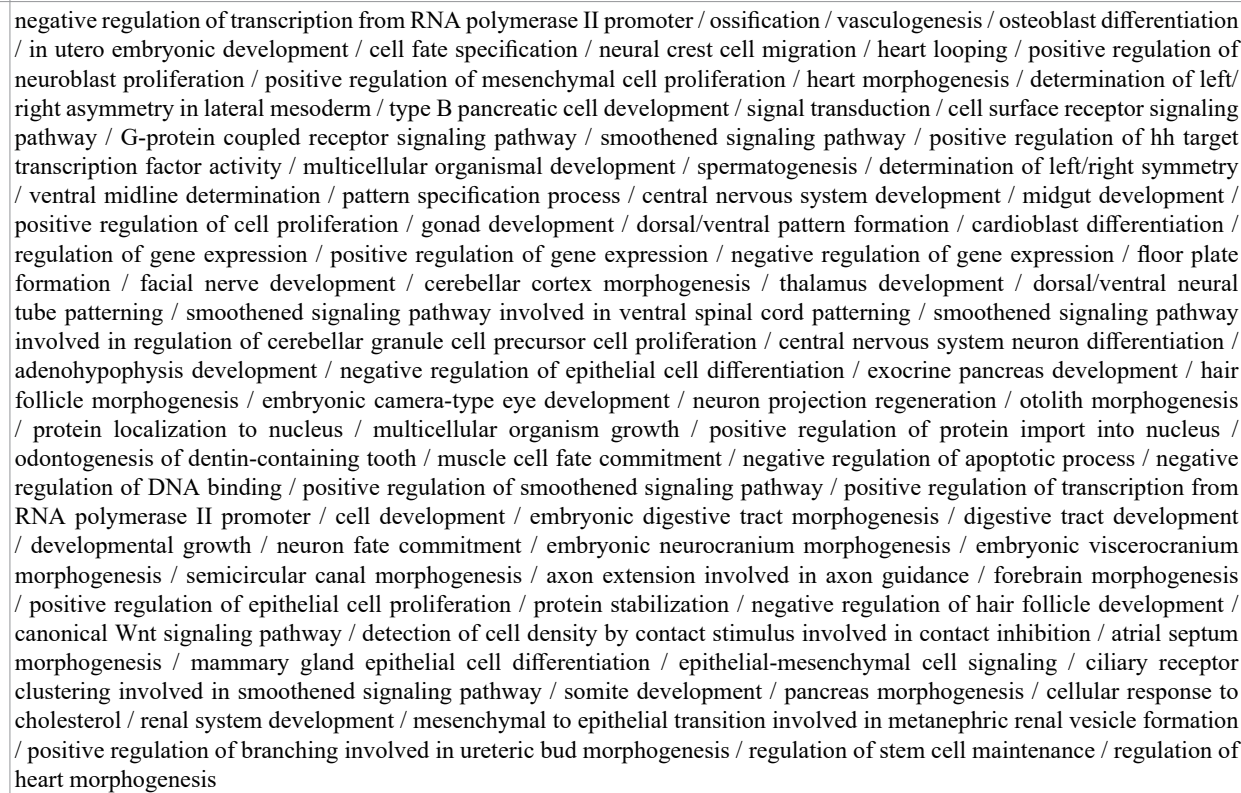 \\
\hline SMURF1 & $\begin{array}{l}\text { SMAD specific E3 ubiquitin protein } \\
\text { ligase } 1\end{array}$ & $\begin{array}{l}\text { protein polyubiquitination / protein export from nucleus / transforming growth factor beta receptor signaling pathway / / } \\
\text { ectoderm development / metabolic process / protein ubiquitination / cell differentiation / negative regulation of ossification } \\
\text { / BMP signaling pathway / negative regulation of transforming growth factor beta receptor signaling pathway / negative } \\
\text { regulation of BMP signaling pathway / ubiquitin-dependent SMAD protein catabolic process / receptor catabolic process } \\
\text { / protein localization to cell surface / protein ubiquitination involved in ubiquitin-dependent protein catabolic process / } \\
\text { proteasome-mediated ubiquitin-dependent protein catabolic process }\end{array}$ \\
\hline
\end{tabular}




\begin{tabular}{|c|c|c|}
\hline SMURF2 & $\begin{array}{l}\text { SMAD specific E3 ubiquitin protein } \\
\text { ligase } 2\end{array}$ & $\begin{array}{l}\text { negative regulation of transcription from RNA polymerase II promoter / transcription, DNA-templated / transcription initiation } \\
\text { from RNA polymerase II promoter / ubiquitin-dependent protein catabolic process / transforming growth factor beta receptor } \\
\text { signaling pathway / metabolic process / gene expression / protein ubiquitination / regulation of transforming growth factor } \\
\text { beta receptor signaling pathway / BMP signaling pathway / negative regulation of transforming growth factor beta receptor } \\
\text { signaling pathway / ubiquitin-dependent SMAD protein catabolic process / protein ubiquitination involved in ubiquitin- } \\
\text { dependent protein catabolic process / negative regulation of transcription, DNA-templated }\end{array}$ \\
\hline SPPL2B & signal peptide peptidase like 2B & $\begin{array}{l}\text { proteolysis / membrane protein ectodomain proteolysis / membrane protein intracellular domain proteolysis / regulation of } \\
\text { immune response }\end{array}$ \\
\hline ST3GAL4 & $\begin{array}{l}\text { ST3 beta-galactoside alpha-2,3- } \\
\text { sialyltransferase } 4\end{array}$ & $\begin{array}{l}\text { carbohydrate metabolic process / protein glycosylation / O-glycan processing / keratan sulfate biosynthetic process / protein } \\
\text { N-linked glycosylation via asparagine / glycosaminoglycan metabolic process / keratan sulfate metabolic process / post- } \\
\text { translational protein modification / cellular protein metabolic process / small molecule metabolic process / cognition / sialylation }\end{array}$ \\
\hline ST3GAL6 & $\begin{array}{l}\text { ST3 beta-galactoside alpha-2,3- } \\
\text { sialyltransferase } 6\end{array}$ & $\begin{array}{l}\text { carbohydrate metabolic process / cellular protein modification process / protein glycosylation / glycolipid metabolic process } \\
\text { / oligosaccharide metabolic process / keratan sulfate biosynthetic process / glycosaminoglycan metabolic process / keratan } \\
\text { sulfate metabolic process / small molecule metabolic process / cellular response to interleukin-6 / sialylation }\end{array}$ \\
\hline ST6GALNAC3 & $\begin{array}{l}\text { ST6 (alpha-N-acetyl-neuraminyl- } \\
\text { 2,3-beta-galactosyl-1,3)-N- } \\
\text { acetylgalactosaminide alpha-2,6- } \\
\text { sialyltransferase } 3\end{array}$ & protein glycosylation / glycosylceramide metabolic process / sialylation \\
\hline ST8SIA4 & $\begin{array}{l}\text { ST8 alpha-N-acetyl-neuraminide } \\
\text { alpha-2,8-sialyltransferase } 4\end{array}$ & $\begin{array}{l}\text { ganglioside biosynthetic process / cellular protein modification process / protein glycosylation / N-glycan processing / nervous } \\
\text { system development / axon guidance / oligosaccharide metabolic process / sialylation }\end{array}$ \\
\hline SULF2 & sulfatase 2 & $\begin{array}{l}\text { kidney development / chondrocyte development / glomerular filtration / metabolic process / positive regulation vascular } \\
\text { endothelial growth factor production / esophagus smooth muscle contraction / positive regulation of Wnt signaling pathway / } \\
\text { heparan sulfate proteoglycan metabolic process / glomerular basement membrane development / glial cell-derived neurotrophic } \\
\text { factor receptor signaling pathway / negative regulation of fibroblast growth factor receptor signaling pathway / embryonic } \\
\text { skeletal system development / cartilage development / bone development / innervation }\end{array}$ \\
\hline TGFBR3 & $\begin{array}{l}\text { transforming growth factor, beta } \\
\text { receptor III }\end{array}$ & $\begin{array}{l}\text { blood vessel development / response to hypoxia / in utero embryonic development / blastocyst development / epithelial to } \\
\text { mesenchymal transition / liver development / heart morphogenesis / protein complex assembly / immune response / transforming } \\
\text { growth factor beta receptor signaling pathway / transforming growth factor beta receptor complex assembly / negative regulation } \\
\text { of epithelial cell migration / negative regulation of epithelial to mesenchymal transition / cell growth / cell migration / BMP } \\
\text { signaling pathway / positive regulation of transforming growth factor beta receptor signaling pathway / negative regulation } \\
\text { of transforming growth factor beta receptor signaling pathway / organ regeneration / response to follicle-stimulating hormone } \\
\text { / response to prostaglandin E / response to luteinizing hormone / intracellular signal transduction / regulation of protein } \\
\text { binding / negative regulation of epithelial cell proliferation / positive regulation of NF-kappaB transcription factor activity / / } \\
\text { negative regulation of cellular component movement / ventricular cardiac muscle tissue morphogenesis / palate development / } \\
\text { cardiac muscle cell proliferation / definitive hemopoiesis / cardiac epithelial to mesenchymal transition / definitive erythrocyte } \\
\text { differentiation / heart trabecula formation / pathway-restricted SMAD protein phosphorylation }\end{array}$ \\
\hline TMEM2 & transmembrane protein 2 & multicellular organismal development \\
\hline TMEM131 & transmembrane protein 131 & --- \\
\hline TMEM132A & transmembrane protein 132A & --- \\
\hline TMEM160 & transmembrane protein 160 & --- \\
\hline TOR1AIP1 & torsin $\mathrm{A}$ interacting protein 1 & positive regulation of ATPase activity / protein localization to nucleus / nuclear membrane organization \\
\hline TPST2 & tyrosylprotein sulfotransferase 2 & peptidyl-tyrosine sulfation / fusion of sperm to egg plasma membrane / prevention of polyspermy \\
\hline TTC17 & tetratricopeptide repeat domain 17 & cell projection organization / actin filament polymerization / cilium organization \\
\hline TXNDC15 & thioredoxin domain containing 15 & cell redox homeostasis \\
\hline UGT3A2 & $\begin{array}{l}\text { UDP glycosyltransferase family } 3 \\
\text { member A2 }\end{array}$ & cellular response to genisterin / flavonoid biosynthetic process / flavonoid glucuronidation \\
\hline UGT8 & UDP glycosyltransferase 8 & $\begin{array}{l}\text { protein localization to paranode region of axon / carbohydrate metabolic process / lipid metabolic process / sphingolipid } \\
\text { metabolic process / galactosylceramide biosynthetic process / cytoskeleton organization / central nervous system development } \\
\text { / peripheral nervous system development / axon cargo transport / metabolic process / lipid glycosylation / paranodal junction } \\
\text { assembly / neuron projection morphogenesis }\end{array}$ \\
\hline WWP1 & $\begin{array}{l}\text { WW domain containing E3 } \\
\text { ubiquitin protein ligase } 1\end{array}$ & $\begin{array}{l}\text { signal transduction / central nervous system development / metabolic process / viral process / protein ubiquitination / } \\
\text { modulation by virus of host morphology or physiology / ion transmembrane transport / protein ubiquitination involved in } \\
\text { ubiquitin-dependent protein catabolic process / proteasome-mediated ubiquitin-dependent protein catabolic process / negative } \\
\text { regulation of transcription, DNA-templated / viral entry into host cell / transmembrane transport }\end{array}$ \\
\hline WWP2 & $\begin{array}{l}\text { WW domain containing E3 } \\
\text { ubiquitin protein ligase } 2\end{array}$ & $\begin{array}{l}\text { negative regulation of transcription from RNA polymerase II promoter / cellular protein modification process / negative } \\
\text { regulation of gene expression / viral process / protein ubiquitination / modulation by virus of host morphology or physiology } \\
\text { / negative regulation of transporter activity / regulation of ion transmembrane transport / regulation of membrane potential / } \\
\text { protein ubiquitination involved in ubiquitin-dependent protein catabolic process / proteasome-mediated ubiquitin-dependent } \\
\text { protein catabolic process / negative regulation of sequence-specific DNA binding transcription factor activity / negative } \\
\text { regulation of transcription, DNA-templated / viral entry into host cell / negative regulation of protein transport / protein } \\
\text { autoubiquitination / protein K63-linked ubiquitination / regulation of potassium ion transmembrane transporter activity }\end{array}$ \\
\hline YAP1 & Yes-associated protein 1 & $\begin{array}{l}\text { vasculogenesis / embryonic heart tube morphogenesis / regulation of transcription, DNA-templated / transcription from } \\
\text { RNA polymerase II promoter / transcription initiation from RNA polymerase II promoter / cellular response to DNA damage } \\
\text { stimulus / cell proliferation / positive regulation of cell proliferation / gene expression / regulation of keratinocyte proliferation } \\
\text { / keratinocyte differentiation / negative regulation of epithelial cell differentiation / notochord development / somatic stem cell } \\
\text { maintenance / hippo signaling / regulation of cell proliferation / negative regulation of apoptotic process / positive regulation } \\
\text { of transcription from RNA polymerase II promoter / positive regulation of organ growth / paraxial mesoderm development / / } \\
\text { lateral mesoderm development / contact inhibition / cellular response to gamma radiation / regulation of stem cell proliferation } \\
\text { / positive regulation of canonical Wnt signaling pathway / negative regulation of extrinsic apoptotic signaling pathway }\end{array}$ \\
\hline
\end{tabular}




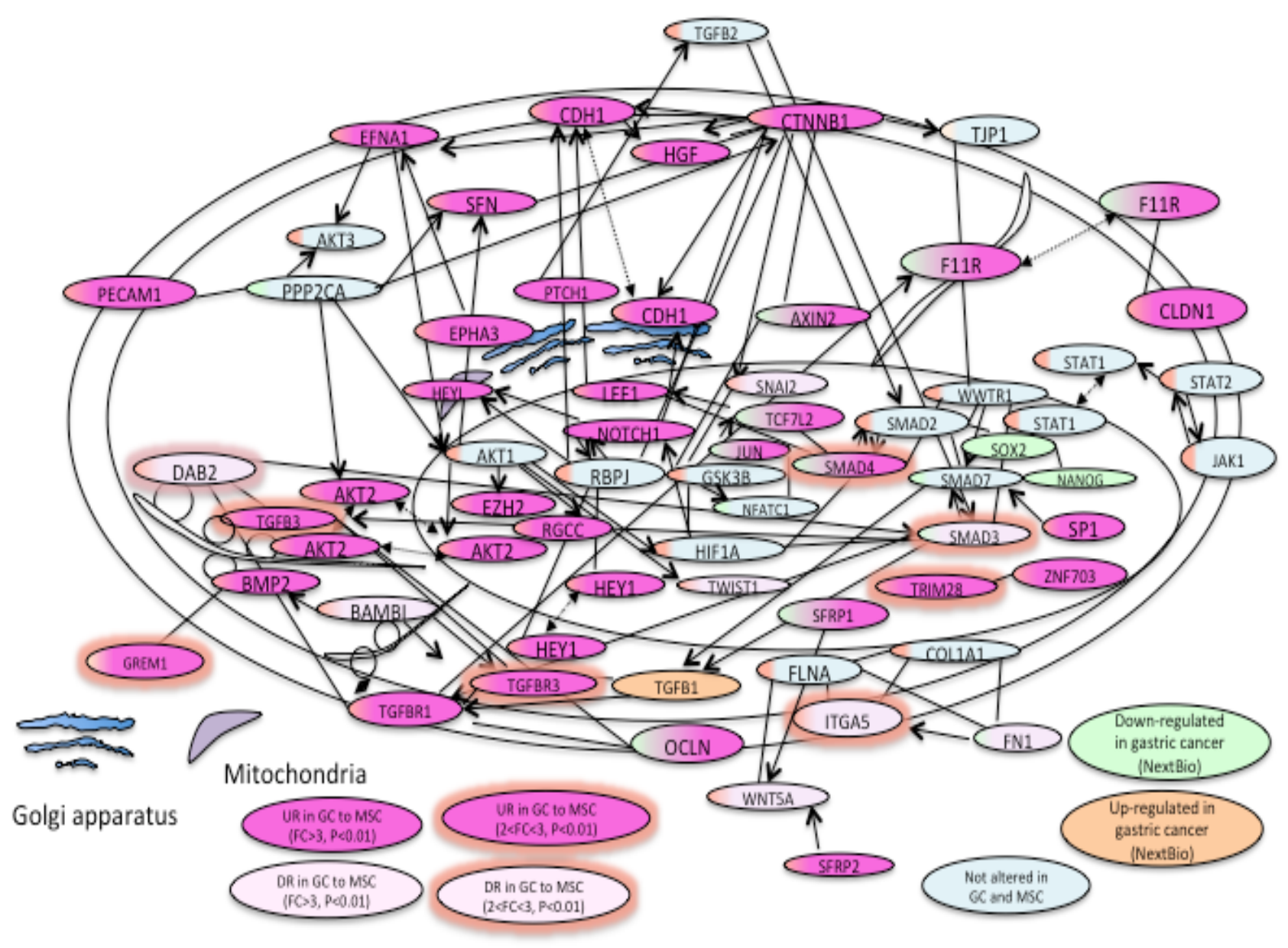

Figure 3. Network model for PTCH1 and EMT-related genes.

Network model for PTCH1 and EMT-related genes are shown. The up-regulated (UR) genes in diffuse-type gastric (GC) compared to mesenchymal stem cells (MSCs) are shown in pink, whereas down-regulated (DR) genes are shown in light purple. Genes with fold change (FC) greater than 3 and $p$ value less than 0.01 are shown. The genes showing FC between 2 and 3 , and $p$ value less than 0.01 are highlighted with beige. UR genes in GC in NextBio database are shown in an orange gradient, whereas DR genes are shown in green gradient. The genes not altered in $\mathrm{GC}$ and MSCs are shown in light blue ( $\mathrm{FC}<2$ or $p>0.05$ ).

interactors in Homo sapiens, which have been further reduced to 88 interactors after subtracting 6 pre-published interactors. Caveolin 1 , caveolae protein, $22 \mathrm{kDa}(\mathrm{CAV} 1)$ and smoothened, frizzled class receptor (SMO) also interact with $\mathrm{PTCH} 1[1,22]$. From the reconstituted complex of purified proteins, PTCH1 was revealed to interact with YES-associated protein 1 (YAP1), which plays an important role in Hippo signalling pathway [23].

\section{Discussion}

The Hedgehog signalling pathway, which is a major network involving PTCH1, is evolutionarily conserved in the development and maintenance of tissues [24]. Expression patterns of PTCH1 are important for stage- and tissue-specific regulation in the Hedgehog response [24]. The human PTCH1 gene plays a role as a tumour suppressor and a developmental regulator [25-27]. Through investigations of mouse Patched mutants, PTCH1 has been revealed to regulate growth and neural pattern formation in the cerebellum and in neural development, which implicates the Hh-PTCH pathway as a potential target for tumour therapies [25]. The previous research studying cyclin-dependent kinase inhibitors in mice heterozygous for Ptch1 has revealed that PTCH1 collaborates with p27Kip1 to prevent medulloblastoma [28]. The investigation of Ptch1 heterozygous mice has also revealed that the loss of Ptch1 subsequent induces senescence, followed by tumour proliferation with p16ink4a inactivation and p53 mutation [29].

A study using zebrafish indicated that the ptch1/2 gene has differential expression during larval head development [30]. The Ptch1 gene regulates morphological formation Lake Malawi cichlids [31]. Further investigations into the connection between tumour suppressive effects and skeletal formation, both regulated by PTCH1, are needed.

It is known that the myeloid cell leukaemia 1 (MCL1), which is overexpressed in cancer, can be inhibited with an MCL1 inhibitor S63845, a small molecule that specifically binds with high affinity to the BH3-binding groove of MCL1. The inhibition of MCL1 results in repressing cancer activity, which implicates this overexpressed protein as a target for future cancer therapies [32]. Considering that PTCH1 is a tumour suppressor and is mutated in several cancers, the upregulation of PTCH1 in diffuse-type GC compared to MSCs may have some importance in terms of targeted therapy.

Immunohistochemistry data has revealed that VIM, a mesenchymal marker, of which gene expression was up-regulated in one probe set and down-regulated in another probe set in diffuse-type GC compared to MSCs, was expressed intratumoral vessels and negative in primary 
Path Designer Network 1

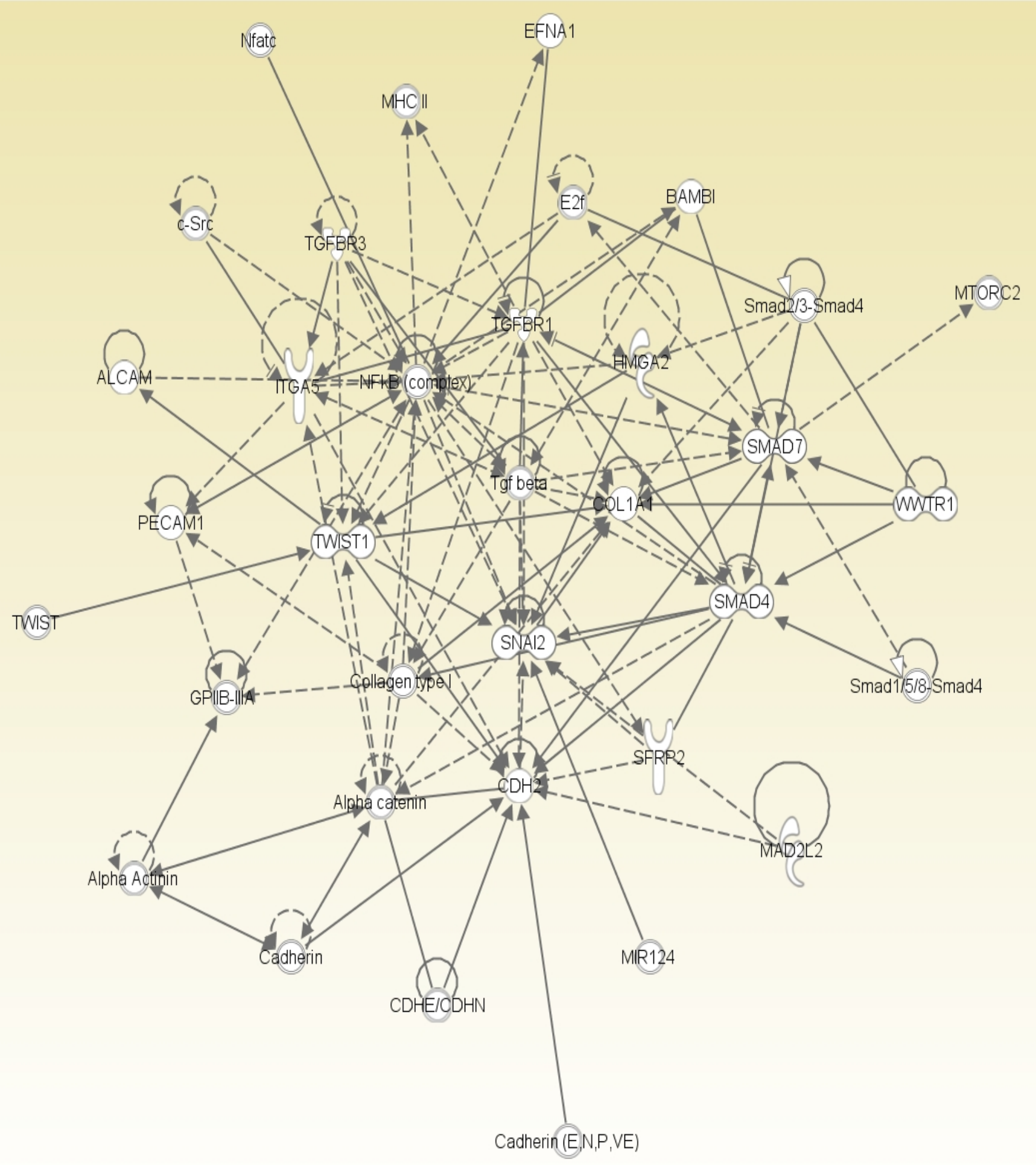

(2) 2000-2018 QIAGEN. All rights reserved.

Figure 4. Network generated by Ingenuity Pathway Analysis (IPA)

A network generated with PTCH1 and EMT-related genes included ALCAM, BAMBI, CDH2, COL1A1, EFNA1, HMGA2, ITGA5, MAD2L2, PECAM1, SFRP2, SMAD4, SMAD7, SNAI2, TGFBR1, TGFBR3, TWIST1 and WWTR1. It was associated with cardiovascular system development and function, and cellular movement. 
lesions in GC [33]. VIM was queried for network analysis in cBioPortal for Cancer Genomics, which resulted in PTCH1 model network generation including VIM.

The metastasis was induced by stemness promotion by HER2, WNT4 or RANKL [34]. The activation of migration was observed in migration/sphere-formation assays [34]. The high-throughput 3D screening system for EMT inhibitors has revealed that TGF-beta receptor I inhibitor SB431542 suppressed the EMT-related phenotypic changes such as mRNA level alteration of $\mathrm{CDH} 1, \mathrm{CDH} 2$, VIM and zinc finger E-box binding homeobox 1 (ZEB1) [35].

A database, such as the National Health and Nutritional Examination Survey (NHANES), which allows the correlation analysis of gene expression, cancer types, and caner stages, and patient demographics and phenotypes, may be useful to understand cancer mechanism and improve public health [36].

In conclusion, the Hedgehog receptor PTCH1 gene expression was found to be up-regulated in diffuse-type GC compared to MSCs in microarray analysis. PTCH1 and EMT-related molecular network was generated, which may reveal the EMT mechanism in diffuse-type GC and stem cells.

\section{Acknowledgements}

We gratefully acknowledge Dr Ryoji Kushima for the pathological and clinical evaluations. We would also like to thank Ms. Rie Komatsuzaki and Ms. Fumiko Chiwaki for their technical assistance. This study was supported in part by the National Institute of Biomedical Innovation, the Ministry of Health, Labour and Welfare of Japan, National Cancer Centre Research and Development Fund, the Ministry of Education, Culture, Sports, Science and Technology of Japan, and the Princess Takamatsu Cancer Research Fund. The authors would like to thank Dr. Akihiko Hirose and Dr. Takashi Yamada for supporting the preparation of the manuscript. The authors would like to thank Prof. Kei Yura for introducing us to the VaProS database. This research is supported by Platform Project for Supporting Drug Discovery and Life Science Research (Basis for Supporting Innovative Drug Discovery and Life Science Research (BINDS)) from Japan Agency for Medical Research and Development (AMED).

Supported by the National Institute of Biomedical Innovation Fund, the Ministry of Health, Labour and Welfare of Japan Fund, National Cancer Centre Research and Development Fund, the Ministry of Education, Culture, Sports, Science and Technology of Japan Fund, and the Princess Takamatsu Cancer Research Fund.

Institutional review board statement: Diffuse-type gastric cancer (GC) tissues were originally provided by the National Cancer Centre Hospital after obtaining written informed consent from each patient and approval from the National Cancer Centre Institutional Review Board (ID: No. 17-030). Existing, publicly available data were analysed in the article.

\section{References}

1. Carpenter D, Stone DM, Brush J, Ryan A, Armanini M, et al. (1998) Characterization of two patched receptors for the vertebrate hedgehog protein family. Proc Natl Acad Sci USA 95: 13630-13634. [Crossref]

2. Chen H, Wang J, Yang H, Chen D, Li P (2016) Association between FOXM1 and hedgehog signaling pathway in human cervical carcinoma by tissue microarray analysis. Oncol Lett 12: 2664-2673. [Crossref]

3. Chen XL, Chinchilla P, Fombonne J, Ho L, Guix C, et al. (2014) Patched-1 proapoptotic activity is downregulated by modification of K1413 by the E3 ubiquitin-protein ligase Itchy homolog. Mol Cell Biol 34: 3855-3866. [Crossref]
4. Mishra P, Panda A, Bandyopadhyay A, Kumar H, Mohiddin G (2015) Sonic Hedgehog Signalling Pathway and Ameloblastoma - A Review. J Clin Diagn Res 9: ZE10-13. [Crossref]

5. Chung JH, Larsen AR, Chen E, Bunz F (2014) A PTCH1 homolog transcriptionally activated by 53 suppresses Hedgehog signaling. J Biol Chem 289: 33020-33031. [Crossref]

6. Li R, Cai L, Hu CM, Wu TN, Li J (2015) Expression of hedgehog signal pathway in articular cartilage is associated with the severity of cartilage damage in rats with adjuvant-induced arthritis. J Inflamm (Lond) 12: 24. [Crossref]

7. Panwalkar P, Moiyadi A, Goel A, Shetty P, Goel N, et al. (2015) miR-206, a cerebellum enriched miRNA is downregulated in all medulloblastoma subgroups and its overexpression is necessary for growth inhibition of medulloblastoma cells. $J \mathrm{Mol}$ Neurosci 56: 673-680. [Crossref]

8. Singh SR (2013) Cancer stem cells: recent developments and future prospects. Cancer Lett 338: 1-2. [Crossref]

9. Tanabe S, Kawabata T, Aoyagi K, Yokozaki H, Sasaki H (2016) Gene expression and pathway analysis of CTNNB1 in cancer and stem cells. World J Stem Cells 8: 384-395. [Crossref]

10. Blokzijl F, de Ligt J, Jager M, Sasselli V, Roerink S, et al. (2016) Tissue-specific mutation accumulation in human adult stem cells during life. Nature 538: 260-264. [Crossref]

11. Tanabe S, Aoyagi K, Yokozaki H, Sasaki H (2014) Gene expression signatures for identifying diffuse-type gastric cancer associated with epithelial-mesenchymal transition. Int J Oncol 44: 1955-1970. [Crossref]

12. Tanabe S, Sato Y, Suzuki T, Suzuki K, Nagao T, et al. (2008) Gene expression profiling of human mesenchymal stem cells for identification of novel markers in early- and latestage cell culture. J Biochem 144: 399-408. [Crossref]

13. Gao J, Aksoy BA, Dogrusoz U, Dresdner G, Gross B, et al. (2013) Integrative analysis of complex cancer genomics and clinical profiles using the cBioPortal. Sci Signal 6 : p11. [Crossref]

14. Cerami E, Gao J, Dogrusoz U, Gross BE, Sumer SO, et al. (2012) The cBio cancer genomics portal: an open platform for exploring multidimensional cancer genomics data. Cancer Discov 2: 401-404. [Crossref]

15. Cancer Genome Atlas Research Network (2014) Comprehensive molecula characterization of gastric adenocarcinoma. Nature 513: 202-209. [Crossref]

16. Stark C, Breitkreutz BJ, Reguly T, Boucher L, Breitkreutz A, et al. (2006) BioGRID a general repository for interaction datasets. Nucleic Acids Res 34: D535-D539. [Crossref]

17. Uhlén M, Fagerberg L, Hallström BM, Lindskog C, Oksvold P, et al. (2015) Proteomics Tissue-based map of the human proteome. Science 347: 1260419. [Crossref]

18. The UniProt Consortium (2017) UniProt: the universal protein knowledgebase. Nucleic Acids Res 45: D158-158D169. [Crossref]

19. Kupershmidt I, Su QJ, Grewal A, Sundaresh S, Halperin I, et al. (2010) Ontologybased meta-analysis of global collections of high-throughput public data. PLoS One 5. [Crossref]

20. Krämer A, Green J, Pollard J Jr, Tugendreich S (2014) Causal analysis approaches in Ingenuity Pathway Analysis. Bioinformatics 30: 523-530. [Crossref]

21. Tanabe S, Aoyagi K, Yokozaki H, Sasaki H (2015) Regulated genes in mesenchyma stem cells and gastric cancer. World J Stem Cells 7: 208-222. [Crossref]

22. Karpen HE, Bukowski JT, Hughes T, Gratton JP, Sessa WC, et al. (2001) The Sonic hedgehog receptor patched associates with caveolin-1 in cholesterol-rich microdomains of the plasma membrane. J Biol Chem 276: 19503-19511. [Crossref]

23. Schuchardt BJ, Mikles DC, Hoang LM, Bhat V, McDonald CB, et al. (2014) Ligand binding to WW tandem domains of YAP2 transcriptional regulator is under negative cooperativity. FEBS J 281: 5532-5551. [Crossref]

24. Lorberbaum DS, Ramos AI, Peterson KA, Carpenter BS, Parker DS, et al. (2016) An ancient yet flexible cis-regulatory architecture allows localized Hedgehog tuning by patched/Ptch1. Elife 5: e13550. [Crossref]

25. Goodrich LV, MilenkoviÄ L, Higgins KM, Scott MP (1997) Altered neural cell fates and medulloblastoma in mouse patched mutants. Science 277: 1109-1113. [Crossref]

26. Hahn H, Wicking C, Zaphiropoulous PG, Gailani MR, Shanley S, et al. (1996) Mutations of the human homolog of Drosophila patched in the nevoid basal cell carcinoma syndrome. Cell 85: 841-851. [Crossref] 
27. Johnson RL, Rothman AL, Xie J, Goodrich LV, Bare JW, et al. (1996) Human homolog of patched, a candidate gene for the basal cell nevus syndrome. Science 272: 1668-1671. [Crossref]

28. Ayrault O, Zindy F, Rehg J, Sherr CJ, Roussel MF (2009) Two tumor suppressors, p27Kip1 and patched-1, collaborate to prevent medulloblastoma. Mol Cancer Res 7: 33-40. [Crossref]

29. Tamayo-Orrego L, Wu CL, Bouchard N, Khedher A, Swikert SM, et al. (2016) Evasion of Cell Senescence Leads to Medulloblastoma Progression. Cell Rep 14: 2925-2937. [Crossref]

30. Pashay Ahi E, Walker BS, Lassiter CS, Jónsson ZO (2016) Investigation of the effects of estrogen on skeletal gene expression during zebrafish larval head development. PeerJ 4: e1878. [Crossref]

31. Roberts RB, Hu Y, Albertson RC, Kocher TD (2011) Craniofacial divergence and ongoing adaptation via the hedgehog pathway. Proc Natl Acad Sci U S A 108: 13194 13199. [Crossref]
32. Kotschy A, Szlavik Z, Murray J, Davidson J, Maragno AL, et al. (2016) The MCL1 inhibitor S63845 is tolerable and effective in diverse cancer models. Nature 538: 477 482. [Crossref]

33. Iwatsuki M, Mimori K, Fukagawa T, Ishii H, Yokobori T, et al. (2010) The clinica significance of vimentin-expressing gastric cancer cells in bone marrow. Ann Surg Oncol 17: 2526-2533. [Crossref]

34. Hosseini H, ObradoviÄ MM, Hoffmann M, Harper KL, Sosa MS, et al. (2016) Early dissemination seeds metastasis in breast cancer. Nature. [Crossref]

35. Arai K, Eguchi T, Rahman MM, Sakamoto R, Masuda N, et al. (2016) A novel highthroughput 3D screening system for EMT inhibitors: a pilot screening discovered the EMT inhibitory activity of CDK2 inhibitor SU9516. PLoS One 11: e0162394. [Crossref]

36. Patel CJ, Pho N, McDuffie M, Easton-Marks J, Kothari C, et al. (2016) A database of human exposomes and phenomes from the US National Health and Nutrition Examination Survey. Sci Data 3: 160096. [Crossref]

Copyright: ( 2018 Tanabe S. This is an open-access article distributed under the terms of the Creative Commons Attribution License, which permits unrestricted use, distribution, and reproduction in any medium, provided the original author and source are credited. 